\title{
LITERATURWISSENSCHAFT
}

\author{
http://dx.doi.org/10.18778/2196-8403.2011.12
}

ANETA JACHIMOWICZ

\section{Der Sieg der „Unform“. Das „Schnee“-Kapitel des Zauberbergs}

W ostatnich dwudziestu latach Czarodziejska górę Tomasza Manna zdominowały dwa sprzeczne kierunki interpretacji. $Z$ jednej strony uznaje się, że powieść ta jest powieścią edukacyjną, z drugiej odczytuje się ją jako powieść filozoficzną o głębokich wpływach schopenhauerowskiej pesymistycznej wizji życia. Celem artykułu jest pokazanie sprzecznych poglądów naukowych na temat powieści, a w szczególności rozdziału „Śnieg“, który przez badaczy dzieła słusznie postrzegany jest jako kluczowy. Wychodząc od różnych i często sprzecznych sposobów odczytywania wizji Castorpa, autorka artykułu stawia sobie za cel zbadanie snu z rozdziału „Śnieg“ przy użyciu freudowskiego instrumentarium. Jeśli sny mamy rozumieć, jak chciał tego Freud, jako ,spełnione marzenia', to narzuca się pytanie, co w takim razie znaczy sen Castorpa, co uosabiają jego wizje, i czy Castorp rzeczywiście zbliża się poprzez swój sen do myśli humanistycznej i przechodzi - jak to wyraził sam Tomasz Mann - przez „program wychowawczy“.

Thomas Manns Roman Der Zauberberg wird in den letzten zwanzig Jahren von zwei polaren Deutungen bestimmt: von der Deutung als Bildungsroman und von der als philosophischem Roman, der der pessimistischen Lebensstimmung Schopenhauers unterworfen sei. Ziel dieses Beitrages ist, die Entwicklungsstufen der wissenschaftlichen Diskussion über den Zauberberg und insbesondere über das Kapitel „Schnee“ zu zeigen, das, vielfach diskutiert, in der Forschung als Schlüssel zum Romanverständnis gesehen wird. Ausgehend von den unterschiedlichen und oft widersprüchlichen Deutungen des Traumes in diesem Kapitel, setzt sich die Autorin des Artikels zum Ziel, das „Schnee-Kapitel“ zur freudschen Traumdeutung in Bezug zu setzen. Dadurch wird versucht, hinter den manifesten Trauminhalten die latenten zu entdecken. Wenn Träume als ,erfüllter Wunsch " aufzufassen sind, so wäre danach zu fragen, was Castorp eigentlich träumt bzw. was die Traumbilder versinnbildlichen und ob sich in Castorp durch den Traum eine Entwicklung zum Humanitätsgedanken hin, ein - so Thomas Mann - „Erziehungsprogramm“ vollzieht. 
During the last twenty years Thomas Mann's The Magic Mountain has been dominated by two contradictory lines of interpretation. On the one hand, it is acknowledged that this tale is a development novel, and on the other, it is interpreted as a philosophical novel, deeply influenced by Schopenhauer's pessimistic vision of life. The aim of this article is to demonstrate the conflicting specialist views on the subject of the story, and in particular the "Snow" chapter which is rightly perceived by researchers of the work as the key one. Starting from various and frequently contradictory ways of interpreting Castorp's vision, the author of the article sets herself the aim of examining the dream from the „Snow“ chapter using Freudian Dream Analysis. If we are to understand dreams as ,fulfilled wishes', as Freud intended, then there raises the question of what Castorp's dream means in this case, what his visions personify and whether Castorp really gets closer to humanist thought through his dream and goes, as Thomas Mann himself put it, through an ,education programme“.

\section{Die Überwindung der Dekadenz}

Im zweiten Teil des Zauberbergs tritt die Handlung in eine neue Phase ein. Hans Castorp experimentiert gedanklich, zeigt Ansätze zu einem individuellen, die Gegensätze überwindenden Denken. Die Stelle der Madame Chauchat - der Personifikation von Eros und Hermes - ersetzt der Jesuit Leo Naphta mit seiner geistesasketischen Ideologie; Mynheer Peeperkorn - die Verbindung von Lebensbejahung und Genusskraft - tritt auf. Höhepunkt des zweiten Teils ist die Humanitätserkenntnis Castorps in der „Schnee“-Vision. Der Wandel in der Buchkonzeption ist erkennbar: Auf den ersten Blick scheinen die Befreiung Hans Castorps von seinem Nihilismus und der Faszination vom Tode die ,intentio operis‘ zu sein.

Dieser Bruch in der Romankonzeption wurde von Thomas-Mann-Forschern als Folge der Entwicklung des Humanitätsgedankens bei Mann erklärt. Seit Anfang der 20er Jahre versuchte der Schriftsteller, sich von der romantischen „Sympathie mit dem Tode“ (LANGER 2009:352) zu lösen sowie den Humanitäts- und Demokratiebegriff in den Mittelpunkt seines Denkens zu stellen. Mann wird nun zum Fürsprecher der Weimarer Republik und zum Kritiker seiner früheren Aufsätze, in denen er den Krieg als Verteidigung deutscher Eigenart rechtfertigte. ${ }^{1}$ Erst unter dem Eindruck des Ersten Weltkrieges und

1 Vgl. KURZKE (2001:237), LANGER (2009:293). Es geht hier insbesondere um den Essay Gedanken im Kriege, der den Krieg als Befreiung aus der Desorientierung pries. Schon in einem Brief an Samuel Fischer vom 22. August 1914 schrieb Mann, dass er die „faulige“ Friedenswelt satt gehabt und dass „ein Gefühl von Reinigung, Erhebung, Befreiung“ ihn ergriffen habe. Den Deutschen habe auch 
der politischen Unruhen der Nachkriegszeit kommt es zur Neuorientierung Manns, die das weitere Schicksal des Romans nach 1921 bestimmen sollten die Abkehr vom Romantischen, vom Pessimismus und von der Dekadenz hin zu einem Bekenntnis zu Demokratie und Republik. ${ }^{2}$ Goethe und Tolstoi, der lebensbejahende Nietzsche und Homer sind nun seine Lehrmeister. ${ }^{3}$

Die Folge der Neuorientierung des Autors lässt sich in der Umkehrung der bisher fallenden Strukturlinie des Romans erkennen. Dem Ausdruck der Todes- und Liebesverfallenheit, die den ersten Teil des Zauberberg bestimmten, stellt Mann nun seinen Humanitätsgedanken gegenüber. Das Umkehren dieser Strukturlinie kündigten zwei politische Schriften an - der Essay Goethe und Tolstoi (1921) und die Rede Von deutscher Republik (1922). Hinter beiden Aufsätzen steckt die Idee, den Roman in einer grundlegend neuen ideellen Richtung fortzusetzen: Hans Castorp soll über Gedankenexperimente von der Eros-Tod-Faszination befreit sowie vom Verfall zur Entwicklung geführt werden. Ins „Schnee“-Kapitel fließen die Schlussgedanken beider Schriften ein. ${ }^{4}$ Der Durchbruch zum Positiven, zum Staat, die Verbindung der Todesromantik und der Lebensbejahung - alles, was im ersten Teil des Romans über die romantische Faszination vom Tod gesagt wurde, wird jetzt auf den Humanitätsbegriff übertragen (LANGER 2007).

nicht Größeres geschehen können, als dass die Welt sich gegen sie erhoben habe (WYSLING / FISCHER 1975:454).

2 Vgl. RieCKMAnN (1979:53). Rieckmann untersucht in seinem Buch wesentliche Elemente der geistigen und politischen Entwicklung Manns in den Jahren 1912 bis 1924. Er betrachtet den Roman als übertragene Autobiographie seines Verfassers. Die Neuorientierung des Autors, über die er in seinen Briefen und Essays reflektierte, beschreibt Rieckmann in Kapitel V seiner Arbeit.

3 Im Juni 1921 wird das fünfte Kapitel des Zauberbergs abgeschlossen. Mann beginnt mit der Arbeit an der Rede Goethe und Tolstoi.

$4 \quad$ Der Schluss des Republikvortrags greift die Synthese von Hans Castorps Traum auf und verkündet sie als Botschaft der Humanität. Die Äußerung „Das Interesse für Tod und Krankheit, für das Pathologische, den Verfall ist nur eine Art von Ausdruck für das Interesse am Leben, am Menschen“ (MANN 1960:851) findet ihre Entsprechung in der Erkenntnis Castorps im „Schnee“-Traum: „Denn alles Interesse für den Tod und Krankheit ist nichts als eine Art von Ausdruck für das [Interesse] am Leben“ (MANN 1991:675). An einer anderen Stelle des Vortrags wird der Wandel in der Romankonzeption des Romans angekündigt: „Es könnte Gegenstand eines Bildungsromans sein, zu zeigen, daß das Erlebnis des Todes zuletzt ein Erlebnis des Lebens ist, daß es zum Menschen führt.“ (MANN 1960:851) 
Bereits seit 1921, einhergehend mit seiner Goethe-Studie, dachte Mann daran, dem Zauberberg in der Nachfolge des Wilhelm Meister den Charakter eines Bildungsromans zu geben. Der Versuch, den Zauberberg als positiven Bildungsroman anzuerkennen, der Hans Castorp letztlich dennoch nicht aus dem Berghof und dem Verfall herausführt, löste einige Verwirrung in der Thomas-Mann-Forschung aus (LANGER 2009:354). Zwar äußert sich der Autor selbst dahingehend über seinen Roman, er sei eine Art Bildungsgeschichte und „Wilhelm Meisteriade“, worin ein junger Mensch durch das Erlebnis von Krankheit und Tod zur Idee des Menschen und des Staates geführt werde $^{5}$, jedoch fehlen die Grundmerkmale der Gattung. Der Protagonist ist keine im Werden begriffene Persönlichkeit, er hat keine gesellschaftlichen Ziele, verhält sich passiv, ergreift keinen Beruf und gründet keine Familie. Der Selbstdeutung des Romans steht die Deutung einiger Forscher gegenüber: Wysling spricht von der „Geschichte der Depersonalisation“ und von einer „Verfallsgeschichte“ (zit. nach KOOPMANN 1995:419), HERMAN KURZKE (1985:210) von einem ,Entbildungsroman“ und BøRGE KRISTIANSEN (1986:55) von einem „Entbildungsprozess“. Darüber hinaus wird betont, dass Mann im Bemühen, Nihilismus und Pessimismus den Menschlichkeitsbegriff entgegenzustellen, daran scheitert, sich von romantischen Tendenzen und der tief in seinem Bewusstsein eingeschriebenen Dekadenz zu befreien. ${ }^{6}$

Im „Schnee“-Kapitel gleicht Hans Castorp seinem Autor. Auch er soll zu humanistischen Einsichten gelangen und über die eigenen Möglichkeiten hinauswachsen. Am Gedanken der Steigerung, d. h. einer positiven, vom Verfall zur Humanität führenden Aufwärtslinie im Roman, kann festgehalten werden, sofern man annimmt, dass Castorp im Traum seine Todessehnsucht endgültig überwindet. Inwiefern sich aber in Castorp eine Entwicklung zum Humanitätsgedanken vollzieht bzw. ob eine Entwicklung überhaupt zustande kommt, diese Frage beschäftigt die Zauberberg-Forscher seit langem. Ein Schlüssel dafür wird im für den Gesinnungswechsel ausschlaggebenden und deutungsvielfältigen ,Schnee“-Kapitel gesucht. Einerseits wird über diesen Traum eine Steigerung bzw. Aufwärtslinie in der Romanstruktur gesehen sowie der Erkenntnissatz der Vision als humaner Höhepunkt des Romans verstanden. Andererseits wird darauf hingewiesen, dass Castorps Traum von der ,schopenhauerischen Strukturlinie“ geprägt sei. Die „Form“ und „Un-

\footnotetext{
5 MANN (1962:199f.) in einem Brief vom 4. September 1922 an Arthur Schnitzler.

6 Vgl. Kristiansen (1986). Mehr dazu im Folgenden.
} 
form“, die den Zauberberg bis zu diesem Moment leitmotivisch bestimmten ${ }^{7}$, ständen sich auch in der Traumvision gegenüber, womit Schopenhauers Verneinung des Willens zum Leben ausgedrückt werde. Eine pessimistische Lebenseinstellung, in Die Welt als Wille und Vorstellung dargelegt sowie Hauptthema der früheren Romane und Erzählungen Manns, verweigere das Lebensmögliche und schließe jede Einwicklung und jede ausgleichende Einsicht aus. Die Befürworter der schopenhauerischen Strukturlinie des Romans sind der Ansicht, die Willensmetaphysik beeinflusse alle Bereiche des Romans.

\section{Die Todeskonfrontation im ,Schnee“-Traum ${ }^{8}$}

Hans Castorp unternimmt im sechsten Kapitel einen abenteuerlich anmutenden Ausflug ins Hochgebirge und hat dort im Traum eine Vision. Castorps Skiwanderung, die Schilderung der Winterwildnis und die ihn begleitenden Gedanken sind eine gesteigerte Wiederholung der Reise zu Beginn des Romans. Das Kapitel enthält zahlreiche Anspielungen auf den ersten Teil des Zauberbergs: Castorp begibt sich ins Unbekannte und Gefährliche, an einen fremden Ort, wie am Anfang des Romans nach Davos. Der neue Gegensatz Tal - Berg steht für den Gegensatz Flachland - Sanatorium. Die Skier, von Settembrini „Flügelschuhe“ (MANN 1991:646) genannt, implizieren die Gestalt des Hermes (KURZKE 1985:204). Wie bereits während seiner Fahrt auf den Zauberberg, verliert Castorp auch beim Aufstieg sowohl Orientierung als auch Zeitbezug, er irrt umher, empfindet ein Angst- und Schwindelgefühl. Nachdem Settembrini bemerkt, dass Castorp ins Bedrohliche strebt, ,ruft [er]

7 Die Begriffe „Form“, „Unform“ (MANN 1991:677) und „Überform“ (MANN 1991:688) sind die Leitmotive des Zauberbergs. Die Form entspricht dem bürgerlichen, nach Normen gerichteten Leben im Flachland. Der Bereich der „Unform“, wird von Castorps Hang zur Bequemlichkeit und absoluten Freiheit, die gegen alle bürgerlichen Normen verstößt, bestimmt. Dieser Bereich tendiert zur Auflösung der Form, die sich angesichts des Willens als Schein erweist. Der Bereich der „Überform“ findet seine Entsprechung in Schopenhauers Philosophie von der Welt als Vorstellung und bedeutet eine Strenge der Form, die keine Nachlässigkeit duldet. Diese drei Bereiche sind einander ausschließende Konstituenten der Identität Castorps.

8 Michael MAar (1995:157) identifiziert die Verführung in der „Schnee“-Welt in Anknüpfung an die Andersen-Märchen Eisjungfrau und Schneekönigin motivisch mit der sexuellen Verführung. In seiner Deutung nimmt die Todeskonfrontation eine sekundäre Stellung ein. 
ihm durch die hohlen Hände eine Warnung nach“ (MANN 1991:648), die mit der Warnung aus der Faschingsnacht zu vergleichen ist. Das „dunstige Nichts" (MANN 1991:651) übt die gleiche Anziehungskraft auf Castorp aus wie Clawdia in der Walpurgisnacht. Die Löcher, die der Skistock im Schnee hinterlässt, erinnern ihn an Clawdias Kirgisenaugen. Castorp befindet sich in „ebenso toller und schlimmer Lage“ wie in jener Nacht, als er „,der kranken Clawdia Chauchat son crayon, seinen Bleistift, Pribislav Hippe's Bleistift zurückgegeben hatte" (MANN 1991:677). Von der Landschaft fühlt er sich ebenso verführt wie von seiner sexuellen Neigung zu Clawdia, ersehnt eine Vereinigung mit ihr und der Natur. Castorps Sympathie für die ,große [...] Winterwildnis“" (MANN 1991:651) ist Ausdruck der Faszination für den Tod wie auch seiner Liebe zu der Russin. Die Hochgebirgswelt ist für ihn das Reich des Todes, in dem das „Urschweigen“, das „Gleichgültig-Tödliche“ (MANN 1991:649), „die schneiende Totenstille“ (MANN 1991:650), das „weiße wirbelnde Nichts“ (MANN 1991:658) herrscht. Das „tote Gelände“ (MANN 1991:655) und die „weißliche Transzendenz“ (MANN 1991:650) erwecken bei ihm „religiöse Furcht“ (MANN 1991:649). Er steht wie bei jeder Konfrontation mit dem Tode ,auf seinen Stock gestützt, den Kopf zur Schulter geneigt, mit offenem Munde“ (MANN 1991:649). Castorp droht „die volle Umarmung" (MANN 1991:649) der tödlichen Natur. Die Schneekristalle, von deren Regelmäßigkeit Castorp entzückt ist, empfindet er als „Geheimnis des Todes“ (MANN 1991:654). In dieser „ringsum gewaltig nichtssagenden Stille“ (MANN 1991:652) durchfahren Erinnerungsfragmente an sein Leben sein Gehirn. Das Pochen seines Herzens erinnert ihn an den Arzt Behrens sowie an die Bilder, die er in der „Durchleuchtungskammer“ gesehen hatte, Naphtas Redensart „Praeteri figura hujus mundi“ (MANN 1991:652) an die tote Landschaft. In der das Sanatorium implizierenden Hütte, die er endlich erschöpft erreicht, trinkt er Portwein - ein ,dilettantische[s] Getränk“ (MANN 1991:666), das er mit dem Kulmbacher Bier vom Abend seiner Ankunft auf dem Zauberberg vergleicht. Der Portwein war „beinahe das Falscheste, was er hätte zu sich nehmen können“ (MANN 1991:666), denn unter seinem Einfluss befreit sich sein Bewusstsein von aller zeitlich-räumlichen Einschränkung, Castorp versetzt sich in einen somnambulen Zustand, sein Traum beginnt. Dieser besteht aus zwei Teilen: einem Bildertraum, der in die einander entgegengesetzten Szenerien von den Sonnenleuten bzw. vom Blutmahl aufgeteilt ist, und einem Gedankentraum, der die Auslegung der Bilder enthält. 
In der ersten Phase seines Traumes sieht Castorp einen Park, von „Heimatodem, Duft und Fülle des Tieflandes“ (MANN 1991:668) erfüllt. Er hört den Gesang eines italienischen Tenors, der ihn in eine südlich-idyllische Landschaft versetzt. Diese Landschaft erinnert ihn an das Mittelmeer, Neapel, Sizilien oder Griechenland. Der Traum ist nicht etwa eine bloße Zusammenfassung des am Tag Erlebten, denn die Traumbilder gehen über den Rahmen seiner Erinnerung hinaus - Castorp hat dieses Mittelmeerparadies nie gesehen. Trotzdem erinnert er sich daran, denn das Paradies aus seinem Traum ist allgegenwärtig. Im Traum erkennt er die menschlichen Urträume, den Paradiesfrieden und das Goldene Zeitalter. Mit dem „Heimatodem", den Buchen und dem Ahorn spielt aber auch Castorps deutsche Heimat in den Traum hinein (WySLING 1995:414). In diesem Arkadien erblickt Castorp die „,verständig-heitere, schöne junge Menschheit“, „Sonnenund Meereskinder" (MANN 1991:670). Sie arbeiten nicht, die Natur ernährt sie. Die Menschen bewegen sich wie Hirten, tanzen und spielen. Castorp ist beeindruckt vom vollkommenen Glück der Sonnenleute, das, wie er bemerkt, von „höfliche[r] Rücksicht und Ehrerbietung“ (MANN 1991:672) im Zeremoniell der Frömmigkeit begleitet wird. Die gleiche Verhaltensweise zeigen die Sonnenleute einer jungen Mutter gegenüber, die ihr Kind stillt. Sie knien vor ihr mit über der Brust gekreuzten Armen und geneigten Köpfen. Castorp entzückt dieses Bild, das er als eine „Mischung von förmlicher Devotion und heiterer Freundschaft“ (MANN 1991:672) wahrnimmt. Der Träumende bemerkt auch einen schönen Knaben, der abseits von seinen Freunden steht. Der Knabe ist eine Personifikation des Hermes Psychagogos, der Castorp mit seinem Blick einer „Todesverschlossenheit“ (MANN 1991:673) in die Welt des Blutmahls in einem mächtigen Tempel versetzt. Im Tempel erblickt der Protagonist unter den Säulen ein Steinbild von Demeter und Persephone. ${ }^{9}$ Hinter dieser Säule tut sich Castorp ein grausames Bild auf - der Träumende erschaut zwei alte Frauen, die in einem blutigen Ritual ein Kind zerreißen:

9 Der Gegensatz zwischen Persephones beiden Leben kann auf die künftige Erkenntnis Castorps hinweisen, dass der Mensch der Herr der Gegensätze sein kann. Die Statuengruppe von Mutter und Tochter ist ebenfalls ein Hinweis auf die Repräsentation des Todes im Tempel und bezeichnet den Punkt des Überganges von der Welt der Lebenden zur Welt der Toten. Die Statue deutet die Hermes-Gestalt an. Der Name Hermes bedeutet ,der vom Steinhaufen', ein Stein ist das Symbol seiner Kraft. Die als Statuen dargestellten Demeter und Persephone führen Castorp in die Unterwelt - in die Welt des Blutmahls. 
Da stand ihm die metallene Tür der Tempelkammer offen, und die Knie wollten dem Armen brechen vor dem, was er mit Starren erblickte. Zwei graue Weiber, halbnackt, zottelhaarig, mit hängenden Hexenbrüsten und fingerlangen Zitzen, hantierten dort drinnen zwischen flackernden Feuerpfannen aufs gräßlichste. Über einem Becken zerrissen sie ein kleines Kind, zerrissen es in wilder Stille mit den Händen [...]. (MANN 1991:674)

Nach diesem grässlichen Bild wacht Castorp auf und legt sich sein „Traumgedicht vom Menschen“ (MANN 1991:677) aus. Er korrigiert seine frühere Auffassung von Leben, Tod und Liebe, versöhnt die in den Gesprächen zwischen Naphta und Settembrini diskutierten Gegensätze von Tod und Leben, Krankheit und Gesundheit, Geist und Natur, auch distanziert er sich von seinen beiden Mentoren, indem er sie „Schwätzer“ (MANN 1991:676) nennt. Der Herr dieser Gegensätze sei der „Homo Dei“ (MANN 1991:676). Castorp nimmt eine mittige Position zwischen „Durchgängerei und Vernunft“, zwischen der „Unform“ und der „Form“ (MANN 1991:677) ein, er definiert den Begriff der Menschlichkeit als Überwindung des Todes durch die Todeserfahrung im Interesse des Lebens:

Wer [...] den Körper, das Leben erkennt, erkennt den Tod. Nur ist das nicht das Ganze [...]. Man muß die andere Hälfte dazu halten, das Gegenteil. Denn alles Interesse für Tod und Krankheit ist nichts als eine Art von Ausdruck für das Leben. (MANN 1991:675)

Der zentrale Gedanke, der seine „Schnee“-Reflexion abschließt, ist der Satz: „Der Mensch soll um der Güte und Liebe willen dem Tode keine Herrschaft einräumen über seine Gedanken." (MANN 1991:677; Hervorhebung im Original) Castorp entscheidet sich für die Liebe zum Leben, die um das wahre Wesen des Todes weiß. Er kommt auch zu der Erkenntnis, dass die Menschen in seinem Traum freundlich und höflich zueinander waren, weil sie wussten, dass der Tod zu ihrem Leben gehört. Ihre Heiterkeit beruht auf dem Wissen um das grauenvolle Blutmahl im Tempel. Castorps Erkenntnis ist die Vorahnung einer Humanität, die alle Gegensätze überbrücken kann. Künftig will er handeln wie die „Sonnenleute“ (MANN 1991:670), d.h. Tod und Leben, Krankheit und Gesundheit sowie Geist und Natur als Einheit betrachten. Im Traum besiegt er seine eigene Faszination von Tod und Krankheit, was eine Voraussetzung für den neuen Humanitätsgedanken ist.

Der „Schnee“-Traum führt zu grundlegend neuen Einsichten. Aber er ist ein Produkt der Ermüdung, der Kälte, des Portweins und „großer Konfusion“ (MANN 1991:638) aufgrund der Naphta-Settembrini-Dispute. Seine im Traum gefassten Beschlüsse vergisst er nach dem Erwachen (MANN 1991:679): „Was er geträumt, war im Verbleichen begriffen. Was er gedacht, 
verstand er schon diesen Abend nicht mehr so recht." Zwar ist Castorps Erkenntnis die Auslegung des Humanitätsgedanken sowie die Wendung zur Menschlichkeit unter Überbrückung aller Gegensätze, aber er vergisst alles, was er im Gedankentraum beschlossen hatte. Sind also das „Schnee“-Kapitel und sein Erkenntnissatz für den Roman ein Wendepunkt? Deutet der Traum eine Aufwärtslinie in der Struktur des Zauberbergs an oder ist er nur ein kurzfristiger Zuwachs in Castorps intellektuellem Vermögen, der aber keine Fortsetzung im Roman findet? Die „Schnee“-Vision bleibt das meistdiskutierte Thema in der Thomas-Mann-Forschung.

\section{Das Kapitel „Schnee“ in den Auseinandersetzungen der Sekundär- literatur}

Den Forschungsstand bestimmen seit ca. 30 Jahren zwei polare Deutungen des Zauberbergs: Die Deutung als Bildungsroman und die Deutung als schopenhauerisch-philosophischer Roman. Die Zauberberg-Forschung der letzten zehn Jahre basiert auf einander entgegengesetzten Deutungslinien, die sich in den 80er und 90er Jahren herausbildeten. Außer nuancierten Lesarten ergaben sich seitdem keine neueren Forschungsergebnisse. Zu nennen wäre an dieser Stelle allerdings die von NeuMANN (2002) herausgegebene Große Kommentierte Frankfurter Ausgabe des Zauberbergs und LANGERs (2009) bei Reclam veröffentlichter Band Erläuterungen und Dokumente zu: Thomas Mann: „Der Zauberberg“, der größtenteils die in der Thomas-Mann-Forschung gängigen Konzeptionen darstellt, ohne dass eine eigene Deutungsmöglichkeit dargelegt wird. ${ }^{10}$ Auch zum „Schnee“-Kapitel erschien bislang keine selbstständige, explizit den Gedankentraum untersuchende Publikation. Ich konzentriere mich also im Folgenden auf die beiden oben genannten Deutungslinien des Romans, die insofern von Bedeutung sind, als ich meine Thesen in der Auseinandersetzung mit ihnen entwickle. Mein Ziel ist darüber hinaus, die bisherigen Lesarten des „Schnee“-Kapitels mit seinen Nuancierungen und in ihrer Komplexität zu ordnen.

Die erste Deutung stützt sich auf Manns eigene Interpretation und betont die steigende Strukturlinie der Entwicklung Hans Castorps, sie sieht seinen Traum als Höhepunkt des Romans. Die Interpretationsprobleme dieser Deu-

10 Vgl. auch LANGER (2007). In den letzten Jahren beherrschten meist der Zeitbegriff und die Musik-Thematik bei Mann die Zauberberg-Forschung. Vgl. dazu u. a. MERTENS (2006) und Hick (2003). 
tung resultieren daraus, dass Castorp seinen Traum vergisst. Der Protagonist verhält sich im nachfolgenden Teil des Romans wieder passiv, setzt die neue Einsicht nicht in die Tat um, tut nichts, um das Duell zwischen Settembrini und Naphta zu verhindern, und wandert im Speisesaal von Tisch zu Tisch, um schließlich an den „schlechten Russentisch“ (MANN 1991:967) zu gelangen. Er empfindet großen „Stumpfsinn“ (MANN 1991:856) sowie „Gereiztheit" (MANN 1991:935) und zieht letztlich in den Krieg, aber nicht, weil er diese Entscheidung selbst trifft, sondern weil er in den Krieg geschickt wird. Einen weiteren Beleg sehen Vertreter dieser Deutung in den oft widersprüchlichen Interpretationen von Mann selbst.

Die zweite Deutung akzentuiert die fallende Strukturlinie des Romans und stellt die These vom Bildungsroman in Frage. Der wichtigste Vertreter dieser Deutung ist Kristiansen, der mit seiner Arbeit eine Interpretation des Zauberbergs vor dem Hintergrund der Schopenhauer-Rezeption bei Mann vorlegt. Kristiansens Deutung wurde von der Forschung, auch jener, die sich gegen die Interpretation als Bildungsroman stellte (Koopmann, Wysling, Kurzke), zumeist abgelehnt. ${ }^{11}$ Sie führte jedoch zu einer Diskussion über das Ziel des Zauberbergs und die Bedeutung Schopenhauers für den Roman (LANGER 2009:371).

Kristiansen wirft der bisherigen Zauberberg-Forschung vor, den Gedankentraum überschätzt bzw. die Vision, über die Castorp zu seiner Erkenntnis gelangt, nicht genug beachtet zu haben. Das habe zu einer einseitigen Deutung des Gedankentraums geführt - zur Deutung als positive, humanitätstragende Antwort des Romans. Kristiansen analysiert die Vision und versucht nachzuweisen, dass Castorps Traum von den Sonnenleuten und dem Blutmahl die schopenhauerische Strukturlinie des Romans mit der Unform und der ihr gegenüberstehenden Form bestätigt. Ins Zentrum seiner Analyse stellt er das Bild der stillenden Mutter, das gleich nach der Beschreibung des utopischen „,blauen Sonnenglücks“ (MANN 1991:670) in einer harmonischen Landschaft folgt. Er glaubt, in der Verhaltensweise der jungen Menschen der Mutter gegenüber Elemente gefunden zu haben, die seine These bestätigen: Diese Episode sei mit den thematischen Zusammenhängen Kirche, Religion und Tod verbunden. Die Lebensform der Sonnenleute stimme mit dem Verhalten

11 WysLing (1988:12) äußerte sich beispielsweise über diese Studie wie folgt: „Der Verfasser arbeitet mit dem hermeneutischen Zirkel und sucht dabei bis zu jener ,Idee' vorzudringen, [...] den ,unbewußten Zusammenhang' [zu finden], der in der Organisation des Werkes wirksam ist.“ 
des formbewussten Joachim Ziemßen überein. Ihre „Kniebeugung, ähnlich dem Kirchenbesucher, der im Vorübergehen vorm Hochalter sich leicht erniedrigt“, ihre „förmliche Devotion“, „Würde und Strenge“ (MANN 1991: 672) deuten auf die leitmotivische Einheit mit Joachims Haltung angesichts von Krankheit und Tod hin. Die Form, die die Sonnenleute repräsentieren, gelte als „Schutzmaßnahme gegen die Wirklichkeit des Todes“ und eher ,als Abwehrreaktion gegen die Dämonie [...] des Blutmahls" (KRISTIANSEN 1986:222). Ähnlich wehre sich die Figur Joachim gegen das „Asiatische“12 in seiner geheimen Liebe zu Marusja. Die Förmlichkeit der jungen Menschen werfe ein dunkles Licht auf die frühere Vision der Sonnenleute. Ihre Gemeinschaft sei der „flachländischen“ (MANN 1974:612) Gesellschaft gleich: Die Form als selbstauferlegter moralischer Zwang hemme zwar die dämonischen Urmächte der humanen Gesellschaft, es komme aber nicht zu einer Übereinstimmung zwischen Formwelt und verdrängter Irrationalität. Diese Deutung der Existenz der Sonnenleute findet ihre Entsprechung in Castorps Erlebnissen auf dem Zauberberg. Er ist zwar im Flachland ein Repräsentant der Welt der Arbeit, der Planung und der Organisation - also der Formwelt -, trägt aber in seinem Herzen die verdrängte Liebe zum Unordentlichen, zur Liederlichkeit und zum Tod (d.h. zur Unform). Sie bricht im Hochland im Kontakt mit Chauchat (auch sie die Unform) aus, so dass die im Flachland mühsam erarbeitete Form verdrängt wird. ${ }^{13}$ Kristiansen kommt zu folgender Schlussfolgerung: Castorp erschaue im Zustand einer mit Schopenhauer verstandenen Somnambulie die Grundbedingungen und Wesensmerkmale menschlicher Existenz, Kultur und Geschichte, also Moral, Würde und Strenge. Die Lebensgemeinschaft der Sonnenleute sei nichts Autonomes, sondern eine Verteidigung, ein Verharren gegen die menschliche Triebhaftigkeit. Seine diesbezüglichen Thesen lassen sich wie folgt zusammenfassen:

12 Das „Asiatische“ (KRISTIANSEN 1986:181) bildet eines der wichtigsten Leitmotive im Zauberberg und repräsentiert den Bereich der Unform im Gegensatz zu dem der Form, die für das bürgerliche, sittliche Leben steht. Das Asiatische Türenwerfen, Fingernägelkauen, sexuelle Ausschweifung und Zeitlosigkeit sind seine Attribute - bedeutet die absolute Freiheit der Sitten in Opposition zu den gesellschaftlichen und zivilisierten Umgangsformen des Westens. Im Roman lässt sich das Asiatische mit der bürgerlichen Welt und ihren Normvorstellungen nicht vereinen, denn es führt zur sozialen Deklassierung und Entbürgerlichung.

13 Als Ausdruck von Castorps Sympathie für die Unform gelten u.a. seine nicht „sonderlich aristokratischen Hände“ (MANN 1991:46) und die Neigung zum Dösen sowie seine Liebe zur Musik. 
- Ein versöhnender Ausgleich zwischen der Formwelt und den verdrängten Mächten des Seins sei nicht möglich,

- als Grundlage für die Formwelt gälten Zwang und moralische Entschlossenheit,

- die formgewordene Wirklichkeit sei immer durch Triebhaftigkeit gefährdet.

„Nicht als Lösung des Form- und Humanitätsproblems, sondern als Hinweis auf die tragische Vergeblichkeit aller Form stellt sich Castorps Bekenntnis zur humanen Gemeinschaft der Sonnenleute letzten Endes dar", resümiert der Literaturwissenschaftler KRISTIANSEN (1986:224). Mit dieser und der folgenden Schlussfolgerung stellte er die bisherigen Lesarten des Gedankentraums in Frage: „Im Rahmen der Schnee-Vision lässt sich der tiefe Pessimismus im Hinblick auf die Möglichkeiten des Menschen und seiner Geschichte kaum überhören.“ (KRISTIANSEN 1986:227)

Kristiansen analysiert den Bildertraum ausführlich und vergleicht die thematischen Leitmotive mit der Existenz Castorps aufmerksam und treffend. Auffallend ist gemäß seiner Auslegung der Zusammenhang der formalen Gemeinschaft der Sonnenleute mit dem bürgerlichen, flachländischen Leben Castorps bzw. mit der Verdrängung seiner Triebhaftigkeit. Kristiansen interpretiert nicht nur die leitmotivischen Rahmen, sondern auch das „Schnee“Kapitel. Seine Interpretation stößt aber hinsichtlich des Gedankentraums auf Deutungsprobleme. Die Vision von Sonnenleuten und Blutmahl ist die Grundlage für Castorps Erkenntnis, denn auf ihrer Basis legt Castorp seinen Traum aus (MANN 1991:676):

Mir träumte vom Stande des Menschen und seiner höfisch-verständigen und ehrerbietigen Gemeinschaft, hinter der im Tempel das gräßliche Blutmahl sich abspielt. [...] Waren sie so höflich und reizend zueinander, die Sonnenleute, im stillen Hinblick auf eben dies Gräßliche? Das wäre eine feine und recht galante Folgerung, die sie da zögen!

In diesem Zitat werden die Vereinbarung und der versöhnende Ausgleich zweier Gegensätze deutlich. Castorp nennt den Menschen den „Herrn der Gegensätze“, der Widerspruch wird überwunden, und er selbst tritt als Individuum auf. „Ich will es mit ihnen halten in meiner Seele“ (MANN 1991:676), postuliert Castorp. Dies ist jedoch kein Bekenntnis zu Settembrinis Formwelt, sondern zur Formwelt der Sonnenleute, die, um das Blutmahl wissend, in ihrer förmlichen Gesellschaft leben. Der Deutung Kristiansens ist vorzuwerfen, dass der Bildertraum nicht im Zusammenhang mit dem Gedanken- 
traum interpretiert wird. Beide Visionen sind zwar die Grundlage für die künftige Erkenntnis, jedoch keine illustrierende Auslegung von Castorps Einsichten.

Ein erheblicher Teil der Zauberberg-Forschung spricht sich für die notwendige Versöhnung der beiden polaren Welten aus, die in der Vision der Sonnenleute und des Blutmahls gezeigt werden. Diese Deutung wird durch das Bild der Duplizität der gegensätzlichen Kunsttriebe des Apollinischen und des Dionysischen aus FRIEDRICH NIETZSCHEs Geburt der Tragödie aus dem Geiste der Musik (1999) gestützt. In Interpretationen wird auf den Zusammenhang zwischen den antagonistischen, aber auch komplementären Visionen des „Schnee“-Kapitels und den scheinbar entgegengesetzten Welten aus der Geburt der Tragödie hingewiesen.

Das Bild des vollkommenen „blauen Sonnenglücks“ steht bei Mann für das Apollinische, mit dem „der Drang zum vollkommenen Für-sich-Sein, zum typischen ,Individuum', zu allem was vereinfacht, heraushebt, stark, deutlich, unzweideutig macht“ (NIETZSCHE 1999:176) zu verstehen ist. Das Apollinische ist die Vernunft und die Schönheit. Dagegen drängt das „Dionysische zu einer Universalität, die alle Extreme umfaßt und alle Grenzen überspielt“ (NIETZSCHE 1999:176). Dionysos ist der Gott des Blutmahls und damit des Todes. Er steht für die Schrecken und Entsetzlichkeiten des Daseins; ihm haben nach NIETZSCHE (1999:35) die Griechen den apollinischen Schönheitsglauben entgegengestellt: „Der Grieche [...], um überhaupt leben zu können, mußte [...] vor sie [,die Entsetzlichkeiten'] hin die glänzende Traumgeburt der Olympischen stellen. [...] Um leben zu können, mußten die Griechen diese Götter, aus tiefster Nöthigung, schaffen.“

Castorps Traum wird von der Darstellung der beiden gegensätzlichen Mächte beeinflusst: Er erschließt sich in seiner Vision sowohl das apollinische Griechenland der Geburt der Tragödie als auch das dionysische Griechenland des orgiastischen Rausches und der Schmerzen. Die Sonnenleute sind wie die Hellenen ,feierlich schreiende [...] Menschen, mit harmonisch tönenden Lauten und rhythmischer Gebärdensprache“ (NIETZSCHE 1999:159). Castorp kennt aber die Grundlage ihrer Freude, denn er weiß, ,wie viel dies Volk leiden [mußte], um so schön werden zu können“ (NIETZSCHE 1999: 159). Die Korrelation der beiden Elemente ist unentbehrlich und gleichermaßen notwendig. Das Dionysische alleine wäre nur ,unzulängliche, chaotische [...] Ausschweifung“, dagegen würde das isolierte Apollinische nur zur „Erstarrungen und Unfruchtbarkeit“ führen (PÜTZ 1999:177). 
Dieser Deutung nach kommt es in Castorps Traum zur Verbindung von der Formwelt der Sonnenleute mit der dämonischen Triebhaftigkeit, was im Gegensatz zu Kristiansens Deutung steht. WYSLING (1988) betont, dass Castorp im Traum die Kategorien von Form, Unform und Überform sprenge, die Kategorien, die ursprünglich das einzige Thema des Romans gewesen seien. Seine Argumentation gegen Kristiansens Auffassung des Traumes ist folgende: Die Urbilder, die Castorp in Traum sehe, gingen über das bisherige Romanschema hinaus. Castorp entscheide sich zwar für die Formwelt der Sonnenleute, aber nicht für die Settembrinis, denn dessen Formbegriff sei aufklärerisch und gehe mit der Arbeit im Flachland einher. In einem anderen Aufsatz bekräftigt WYSLING (1995a:55f.) seine bisherige These:

Castorps mythischer Traum [hebt] den ganzen Roman aus den Angeln und [gibt] ihm neue Koordinaten. [...] Mit den Kategorien Vernunft und Leidenschaft, Form und Unform, die den ersten Teil bestimmt haben - Castorp im Widerstreit zwischen Settembrini und Chauchat - ist es plötzlich nicht mehr getan.

Eine gegensätzliche Position zu Kristiansen vertritt auch JENDREIECK (1977:333), der auf die Analogie zwischen den Sonnenleuten und den Griechen aus der Geburt der Tragödie hinwies. Ihr Blick, ihre universale Humanität sei das Resultat der Versöhnung des Apollinischen mit dem Dionysischen, also der Heiterkeit angesichts des Todes: „Der Erfahrungsgrund dieser Humanität ist der Tod, ihr Gesetz das Wissen um die in Krankheit und Tod sich erschließende universale Identität des Humanen.“

Unmittelbar nach dem Bildertraum denkt Castorp an die beiden Pädagogen Naphta und Settembrini. Die im Traum gewonnene Erkenntnis verhilft ihm dazu, die einseitigen und antithetischen Weltbilder Naphtas und Settembrinis zu überwinden. PÜTZ (1995:262) weist darauf hin, dass die Polarität des Apollinischen und des Dionysischen aus der Geburt der Tragödie die Grundlage für Castorps Distanz den beiden Mentoren gegenüber sei. Castorp bezeichnet sie als „Schwätzerchen“ (MANN 1991:676) und verabschiedet sich von der Dialektik seiner Erzieher: „Ihr Streit und ihre Gegensätze sind selbst nur ein guazzabuglio und ein verworrener Schlachtenlärm, wovon sich niemand betäuben läßt, der nur ein bißchen frei im Kopfe ist und fromm im Herzen." (MANN 1991:676) Den Alternativen Tod oder Leben, Krankheit oder Gesundheit stellt er die Frage entgegen (MANN 1991:676): „Sind das wohl Widersprüche?“ Castorp fühlt sich von nun an als „Herr der Gegensätze“ (MANN 1991:676). Auch wenn er den Erziehern intellektuell nicht folgen kann, verhält er sich ihnen gegenüber geringschätzig. In der Absage an die Diktatur der Widersprüche bringe Castorp seinen Humanitätsgedanken zum 
Der Sieg der „Unform“. Das „Schnee“-Kapitel des Zauberbergs

Ausdruck (PÜTZ 1995:262; vgl. auch RIECKMANN 1979:80 und WYSLING 1995:416).

Die auf der Korrelation des Apollinischen und Dionysischen gestützte Interpretation des „Schnee“-Traumes übermittelt das Positive und Optimistische im Gegensatz zur fallenden Strukturlinie der Deutung bei Kristiansen. Es muss aber betont werden, dass weder Wysling noch Kurzke die Idee einer Steigerung im Zauberberg vertreten. Wysling akzentuiert in seinen Deutungsversuchen die neue Auffassung von Liebe bei Castorp. Die Liebe, als Erkenntnis aus dem Traum hervorgebracht, habe nichts mit der Liebe als Lust zu tun. Dies unterscheidet sich von der Meinung Kristiansens, für den die Liebesauffassung der Hauptfigur auch nach dem „Schnee“-Traum, gemäß der fallenden Strukturlinie des Romans, als Lust und Unform zu verstehen sei. Laut Wysling werde der Begriff der Liebe, der den Roman bisher bestimmt hatte und die Verfallenheit an Eros, erotische Verschmelzung und Wollust implizierte, durch Liebe als Humanität ersetzt. Diese Liebe geht über den leitmotivischen Rahmen hinaus. Der Anblick des Blutmahls lehrt Castorp, dass Tod und Liebe ,ein schlechter Reim, ein abgeschmackter, ein falscher Reim“ (MANN 1991:677) sind. Durch diese Erklärung nimmt Castorp Abschied von seinen Überzeugungen und wendet sich dem Humanitätsgedanken zu. Das scheint nach WYSLING (1995:415) die Aufwärtslinie des Romans zu bestätigen, ,aber Castorp vermag die neue Einsicht nicht in die Tat umzusetzen. Sie vermittelt keine Handlungsmaximen, kein politisches Programm“.

KURZKE (1985:205) dagegen stützt sich auf Kristiansens Analysen, findet aber die Erkenntnis aus dem Gedankentraum ,unter der schopenhauerischen Voraussetzung abstrakt und folgenlos, bloße Meinung“. Der Roman zeige nicht genug Ansätze, um eine konsequente steigende Strukturlinie zu begründen, obwohl der zentrale Satz des Gedankentraumes einen humanistischen Gehalt aufweist. Die Unvollkommenheit des Gedankentraumes bestehe darin, dass Mann ihn als Folge der Wendung zur Republik verfasste, „tief innen aber herrschen weiter die unsittliche ,Sympathie mit dem Tode', die Liebe zum Nirwana, die Neigung zum süßen Schlaf" (KURZKE 1985:205).

Ein starkes Argument für eine fallende Romankurve ist, dass Castorp seinen Traum nach dem Erwachen vergisst. Das Vergessen wirft die Frage auf, ob der Traum überhaupt einen Wendepunkt im Leben Castorps bzw. eine lebenswichtige Entscheidung für den Helden darstellt. JOSEPH ERKME (1998:212) äußert sich diesbezüglich folgendermaßen: 
Es besteht kein Zweifel, dass Hans Castorps Vergesslichkeit am Ende des Schnee-Kapitels - im Sinne Nietzsches - als Schutzvorkehrung zu verstehen ist, die der ruhig-ungestörten ,Einverseelung' dient. Was er geträumt, was er gedacht hat, tritt im Zustande der geistigen Verarbeitung nicht in sein Bewusstsein.

Castorps Vergessen ähnelt dem, was NIETZSCHE (1980:291) als ,aktive Vergesslichkeit“ definierte. Wenn Castorp seinen „Schnee“-Traum vergisst, so ist das nach NIETZSCHE (1980:291) als positive „Verdauung“ zu verstehen. Vergesslichkeit gilt als notwendiges Zubehör der Gesundheit, nur so kann Castorp seine Erlebnisse in der Tiefe verdauen. NEUMANN (2001:78) sieht in Castorps Verhalten Peeperkorn und Clawdia gegenüber einen Beleg dafür, dass er die Erkenntnis des „Schnee“-Traumes nicht vergisst, auch wenn er sie nicht verwirklichen kann, denn der Bildungsroman geht am Ende in den Zeitroman über.

Die Vision der Sonnenleute und ihr durch das Blutmahl gestörtes Glück wird in der Forschung als Bild der Humanitätsidee Manns dargestellt. Vertreter dieser Auffassung sind Helmut Koopmann, Jürgen Scharfschwerdt, Eberhard Hilscher, Inge Diersen und Antal Mádl. Der Schwerpunkt dieser Interpretation des Humanitätsgedanken wird jedoch unterschiedlich gesetzt. KoOPMANN (1988:75) versteht Castorps Aufwachen als ,symbolisch zu interpretierendes Aufwachen zur Erkenntnis nach einem spirituellen Untergang“, also ein Aufwachen aus dem Todesschlaf. Castorp wache zur „Aufklärung und Erleuchtung“, zum „Zu-Sich-Kommen“ (KoOPMANN 1988:75), also zum Leben, auf. Dies sei die letzte Stufe der Initiation Castorps zum Humanismus. Koopmann legt den Schwerpunkt der Humanitätsidee auf Castorps Aufwachen. Er sieht in dem ,Schnee“-Traum und im Aufwachen der Hauptfigur einen Entschluss zum Ich. Diese Auslegung wird auf das Bekenntnis Manns zu neuen Werten, die im Gegensatz zur Romantik, zur Todessympathie stehen, gestützt. Aber das Bekenntnis zur Aufklärung bedeutet nach Koopmann nicht die endgültige Absage an die Romantik und die konsequente Verdrängung des Todes, sondern die durch aufklärerische Werte wie Mitte, Liebe und Güte (MANN 1991:677) vollzogene Überwindung der Todeseuphorie. Diese Deutung findet ihre Widerspiegelung in den Worten Castorps im Gedankentraum. Darüber hinaus betont Koopmann, Mann habe sich mit dem Bekenntnis zur Aufklärung von der Todesmetaphysik der Philosophie Schopenhauers distanziert. Diese Kritik an Kristiansens Darstellung, die alle Bildungsergebnisse im Zauberberg in Abrede stellt, führte zur Diskussion zwischen den beiden Literaturwissenschaftlern. Auch wenn Koopmann und Kristiansen sich hinsichtlich des Umfanges der Schopenhauer-Rezeption im Roman einig sind, kommt KOOPMANN (1988:61-88) zu dem Schluss, dass 
sich Mann der Metaphysik Schopenhauers bedient habe, um vor ihr zu warnen, und nicht, um sie zu verteidigen. ${ }^{14}$

Als Bestandteil der Aufklärung ist neben Liebe und Humanität der Begriff der „Mitte“ zu verstehen. Castorp entscheidet sich aufgrund seiner Erkenntnis für diese Position: „In der Mitte ist des Homo Dei Stand - inmitten zwischen Durchgängerei und Vernunft“ (MANN 1991:676). Castorp sieht in den dialektisch-radikalen Lehren der Pädagogen Settembrini und Naphta keine positive Lösung. Weder Settembrinis Liberalismus noch Naphtas mittelalterlicher Totalitarismus führen zum Glück der Menschheit. KoOPMANN (1988:83) sieht im Begriff der „Mitte“ einen weiteren Leitbegriff des Humanitätsgedankens im Zauberberg. Die Idee der Mitte bedeute nicht nur, eine Synthese aus Gegensätzen zu vollziehen, sondern vor allem, sich als Individuum anzuerkennen. ${ }^{15}$

Mit der Darlegung der verschiedenen, zum Teil gegensätzlichen und einander ausschließenden Deutungen des „Schnee“-Kapitels sollte gezeigt werden, dass die geschichtlich-philosophisch gerichteten Deutungen von Castorps Traum nicht immer vollständig sind und in Bezug auf die Gesamtstruktur des Romans scheitern. Sowohl die in der Forschung behauptete Abhängigkeit von der Philosophie Schopenhauers als auch der Einfluss Nietzsches auf die Struktur der Traumbilder ist trügerisch und wird überschätzt, jedenfalls dann, wenn der Zauberberg als systematische Widerspiegelung dieser Philosophien verstanden wird. In vielen Interpretationen wird ein eindeutig positiver

14 Kristiansen (1985:135-144) ließ diese Kommentare nicht ohne Antwort.

15 MÁDL (1980:74) weist darauf hin, dass sich Castorps Humanitätsidee aus seiner Mediokrität ergebe. Darunter sei zu verstehen, dass Castorp als Durchschnittsmensch zwischen den extrem gegensätzlichen Meinungen seiner Lehrer eine mittlere Stellung finden und einem neuen Humanismus folgen soll. Das Mittel der „Mitte“ sei die Ironie. Dank ihr werden die polaren Meinungen der Pädagogen entschärft und eine Synthese ermöglicht. Im Zusammenhang mit der Idee der Mitte ist Nietzsches Vorstellung vom großen Mittag nicht ohne Belang. Auf diese Parallele wies bereits PÜTZ (1978:141) hin: Bei Nietzsche sei der ,große Mittag" die Zeit der Krisis und des Übergangs, in der eine Wende sichtbar werde. Als Castorp seinen Ausflug in die Alpenwelt unternimmt, ist es zwar schon „Nachmittag um drei“ (MANN 1991:653), aber die Mittagszeit ist noch nicht lange vorbei. Die Patienten halten noch Mittagsruhe, und im Hochgebirge herrscht „Totenstille“ (MANN 1991:650), „bodenloses Schweigen“ und „wattige Lautlosigkeit" (MANN 1991:649). Castorp wird in die Krise gestellt und entscheidet sich für die Position der „Mitte“, die seine früheren Einsichten in Frage stellt. 
Romanschluss gesehen, der das Positive in der Entwicklung Castorps erst bestätigen würde. Castorp sollte nach seinem Traum die Initiative ergreifen, aus dem Berghof ausbrechen und im Flachland eine tätige Existenz führen. In seiner Rückkehr ins Sanatorium und im Vergessen des Traumes wird unterdessen die Beendigung seiner Entwicklung und der Beginn der Zeitverlorenheit gesehen. Sogar die Vertreter des zukunftsorientierten, aus dem Gedankentraum hervorgebrachten Humanismus scheitern bei dem Versuch, den Aufenthalt Castorps auf dem Zauberberg als geistige Entwicklung hin zur Humanität anzuerkennen.

Die beiden gegensätzlichen Positionen der Zauberberg-Kritik scheinen also dem Roman nicht angemessen zu sein. Im Folgenden untersuche ich den „Schnee“-Traum in Bezug auf die früheren Träume Hans Castorps auf dem Zauberberg. Damit soll aufgezeigt werden, warum Castorp von den Sonnenleuten und dem Blutmahl träumt, was die beiden Visionen versinnbildlichen und warum Castorp seinen Traum, vergisst".

\section{Castorps Träume und Freuds Traumtheorie}

Der „Schnee“-Traum ist nicht Castorps erster Traum auf dem Zauberberg. Seit dem ersten Tag im Berghof träumt er sehr oft und lebhaft. Gewisse Anlagen zum Träumen bringt Castorp schon vom Flachland mit, was sich in seiner Vorliebe zur Unform äußert: Die Neigung zum Dösen „mit schlaffem Munde und ohne einen festen Gedanken“ (MANN 1991:44), die Vorliebe für Musik, Maria-Mancini-Zigarren und Bier. Während seine ersten Träume aus den Zusammenstellungen der neuen Eindrücke auf dem Berghof bestehen, so bringen ihn die späteren Träume zu bedeutsamen psychologischen Einsichten. Nach dem verwirrten Traum der ersten Nacht folgt der nächste, kristallisiertere und geschlossenere, dessen Helden Settembrini, Chauchat, Hofrat Behrens und Dr. Krokowski sind. Im dritten Traum begegnet er seinem Schulkameraden Hippe, der seiner Erinnerung und seinem Bewusstsein entglitten war. Hier stellt Castorp eine Ähnlichkeit zwischen Hippe und Chauchat fest. Der Traum bedeutet einen Wandel für Castorp, denn er kommt zu der Erkenntnis, dass Hippe die Quelle seiner Todessympathie sei. Im Anschluss bekommt er Fieber und seine Krankheit bricht aus. Auch das Walpurgisnacht-Erlebnis ist eine Art Tagtraum. Diese vier einzelnen „Traumkomplexe“ (SCHARFSCHWERDT 1967:142) zeugen von einer gewissen Steigerung des intellektuellen Vermögens Castorps. Sie bereiten den Grund für den „Schnee“-Traum, in dem sie ihren Höhepunkt finden. Was ist der Grund 
dafür, dass Castorp gerade im Traum zu neuen humanen Erkenntnissen gelangt, zu Einsichten, die er im Wachzustand nie hätte fassen können? Im Halbschlaf gibt er eine teilweise Antwort auf diese Frage, wenn er sich selbst über seinen Traum äußert (MANN 1991:675):

Man träumt nicht nur aus eigener Seele [...], man träumt anonym und gemeinsam, wenn auch auf eigene Art. Die große Seele, von der du nur ein Teilchen, träumt wohl mal durch dich, auf deine Art, von Dingen, die sie heimlich immer träumt.

In der Thomas-Mann-Forschung untersucht man Castorps Träume aus der Perspektive dreier philosophischer Traumtheorien, der von Schopenhauer, Nietzsche und Freud. Alle drei Traumtheorien sind für das „Schnee“-Kapitel ein berechtigtes Deutungsinstrumentarium, wobei die psychoanalytische Traumdeutung, die FREUD (1973) in seiner Studie darlegte, am aufschlussreichsten ist, auch aus dem Grunde, dass Mann selbst sich intensiv mit der in dieser Abhandlung dargestellten Traumtheorie beschäftigte und von ihr inspirieren ließ. ${ }^{16}$

Freud hatte zeitlebens große Ehrfurcht vor Dichtern. Er suchte die Übereinstimmung zwischen Literatur und den Ergebnissen der Psychoanalyse, daher untersuchte er auch die fiktiven Träume erfundener Helden psychoanalytisch. Freud war der Meinung, dass Autoren der Überzeugung folgen, das Denken und Fühlen der Menschen setze sich im Schlaf fort. Sie ließen Figuren träumen, versuchten, durch deren Träume die Seelenzustände ihrer Protagonisten zu schildern (FREUD 1973:90). Die Gradiva-Analyse aus dem Jahre 1907 gilt als die erste selbstständige Interpretation eines literarischen Werks, durch die Freud seine Traumtheorie mit einem dichterischen Beispiel belegt. ${ }^{17}$

16 MANFRED DieRKS (1991:111-137) hat bereits belegt, dass sich Mann intensiv mit Freuds Gradiva-Analyse beschäftigte. Ziel seines Beitrages war, die bewusste Anlehnung Manns an den Text von Jensen und Freud zu zeigen. Er zeigte die Textechos aus Jensen und Freud im Zauberberg und wie Mann sich ab 1911 aus Jensen und Freud die Technik der Verdichtung aneignete. Dabei konzentrierte sich Dierks auf die Analogien zwischen Jensen und Freud und dem „Hippe“Traum aus dem Zauberberg, ohne dabei den „Schnee“-Traum analysiert zu haben.

17 Zehn Jahre später analysierte Freud eine Kindheitserinnerung aus Dichtung und Wahrheit, dann E.T. A. Hoffmanns Sandmann, bis er sich schließlich mit dem Text Dostojewski und die Vatertötung befasste (URBAN / CREMERIUS 1973:8). 
Die Erzählung Gradiva handelt von der unterdrückten Kindheitsliebe eines jungen Altertumsforschers, die sich in seinen Phantasien und Träumen allmählich wieder durchsetzt. Der Archäologe Norbert Hanold folgt einem unbekannten Zug und fährt nach Süden, nach Pompeji. Dort trifft er das früher geliebte Mädchen Zoë, das er anfänglich für ein Mittagsgespenst, für eine vom Hades zurückgekehrte Pompejanerin aus der Zeit des Vulkanausbruchs hält. Dann jedoch erfährt er die Wahrheit und ergibt sich der Liebe. Freud interpretiert die Novelle mit Rückgriff auf seine seit sechs Jahren vorliegende Traumdeutung. Seine Absicht war, die Leistungsfähigkeit der Psychoanalyse in Hanolds Wahn bzw. seinen Träumen nachzuweisen und damit den Autor, der den Traum intuitiv den psychoanalytischen Einsichten gemäß in seinen Text einwebt, als Verbündeten seiner Theorie zu gewinnen. Freuds Interpretation der Erzählung beginnt mit dem Konflikt der Hauptfigur. Hanold hatte seine erotischen Gefühle verdrängt, die seit der Kindheit an die Figur der Zoë gebunden sind. Als er in einer Sammlung ein viele Jahrhunderte altes Relief mit einem reizvoll schreitenden Mädchen entdeckt, das er Gradiva (die ,Vorschreitende') nennt, fühlt er sich vom Bild aus ihm unbekannten Gründen angezogen. Nun kehren die verdrängten Gefühle zurück und kämpfen gegen die sie verdrängende Macht - gegen Hanolds Interesse für die Altertumswissenschaft. Dieser Kampf zeigt sich an Hanolds Vorstellung, dass das Mädchen aus dem Reliefbild in Pompeji gelebt habe und nun aus dem Hades zurückkehre. Hanold reist nach Pompeji, um dort in der Asche die Spuren der Gradiva zu finden. Zugleich werden in den Träumen des Archäologen die aufsteigenden Erinnerungen an seine Kindheitsliebe durch zahlreiche Entstellungen und Umwandlungen der antiken Welt angepasst, wodurch Zoë, die Hanold nach vielen Jahren zufällig in Pompeji wiedertrifft, für ihn zu Gradiva wird. Sie spielt also die Rolle, die ihr sein Wahn zugeschrieben hat.

Eines der wichtigsten psychoanalytischen Konzepte Freuds ist die Theorie von der Verdrängung eines als bedrohlich empfundenen Triebwunsches. Die Verdrängung ist eine Art Vergessen, das „nicht mit dem Untergang, dem Auslöschen der Erinnerung zusammenfällt" (FREUD 1973:113). Unter dem Einfluss äußerer Einwirkungen entstünden also aus der Erinnerung verschiedene „Verwandlungsprodukte“ und „Abkömmlinge“ (FREUD 1973:113) in Form von verzerrten Bildern, die erst dann verständlich seien, wenn man sie auf das verdrängte Gefühl beziehe. Freud untersuchte die Erzählung Gradiva hinsichtlich seiner Theorie von Verdrängung und Wiederkehr unterdrückter Triebansprüche, wobei er jedes Detail der Erzählung seiner Theorie unterord- 
Der Sieg der „Unform“. Das „Schnee“-Kapitel des Zauberbergs

nete. In Hanolds Leben entsteht ein Konflikt zwischen den zurückkehrenden Kindheitsgefühlen und der sein Gefühl verdrängenden Altertumswissenschaft. Die Stationen der Reise nach Süden bis in die Stadt Pompeji repräsentieren den Prozess der Wiederkehr des Verdrängten. In Pompeji - dem Symbolort der unterdrückten, zugeschütteten Triebe ${ }^{18}$ - erinnert sich der Archäologe an seine Kindheitsliebe und fügt sich in die Realität.

Wenn man Castorps Träume psychoanalytisch interpretiert, so lassen sich Zusammenhänge mit den grundlegenden, in der Gradiva-Analyse dargelegten Konzepten der Traumdeutung feststellen. In meiner Untersuchung geht es jedoch nicht darum, den Zauberberg Manns mit Jensens Gradiva zu vergleichen, sondern die auf die Erzählung angewandte Traumtheorie Freuds auf die Träume Castorps, besonders auf den „Schnee“-Traum, zu übertragen. Der Prozess der „Wiederkehr des Verdrängten“ (FrEUD 1973:134) bildet die Kernstruktur des erotischen Motivs im Zauberberg. Die unterdrückte Homosexualität wird auf die Liebe zu Madame Chauchat, die Castorp an seinen Schulkameraden erinnert, übertragen. Das Verdrängte bricht plötzlich auf, denn das Unbewusste kenne weder Zeit noch Raum und sei immer präsent (FREUD 1992:138). In einem seiner Träume antwortet Castorp auf die Frage, was Zeit ist: „,nichts anderes, als einfach eine stumme Schwester, eine Quecksilbersäure ganz ohne Bezifferung“ (MANN 1991:128). Für das Unbewusste hat die Zeit kein absolutes Maß, das Verdrängte kann jederzeit wiederkehren. In der Hippe-Chauchat-Episode hält sich Mann eng an die Psychoanalyse Freuds, die unterdrückten Triebe Castorps weisen Parallelen zu den verdrängten Triebansprüchen Hanolds in der Erzählung Gradiva auf.

Unbewusst ahnt Hans Castorp schon kurz nach seiner Ankunft, dass etwas in ihm wiederkehren will. Verdrängte Liebe sowie Fieber brechen auf dem Zauberberg aus, denn Liebe und Fieber sind zwei Seiten derselben Neigung seiner unterdrückten Todessympathie. Beim Anblick Madame Chauchats bemerkt er gleich, „daß sie breite Backenknochen und schmale Augen hatte... Eine vage Erinnerung an irgend etwas und irgendwen berührte ihn leicht und vorübergehend, als er das sah..." (MANN 1991:108). Durch die Ähnlichkeit werden alte Gefühle und Erinnerungen an eine Person evoziert,

18 „Es gibt wirklich keine bessere Analogie für die Verdrängung, die etwas Seelisches zugleich unzugänglich macht und konserviert, als die Verschüttung, wie sie Pompeji zum Schicksal geworden ist, und aus der die Stadt durch die Arbeit des Spatens wieder erstehen konnte.“ (FrEUD 1973:118) 
so dass das Verdrängte langsam zurückkehrt. An wen aber Clawdia Chauchat Hans Castorp erinnert, wird erst in seinen Träumen veranschaulicht.

Im zweiten Traum, den Castorp auf dem Zauberberg träumt, versetzt er sich auf den Schulhof und bemerkt Madame Chauchat, von der er sich einen Bleistift leiht. Und da, ,als sie ihn ansah, mit ihren schmalen blaugraugrünen Augen über den breiten Backenknochen“ (MANN 1991:127), erkennt er in Clawdia seinen Schulkameraden Hippe, zu dem er vor Jahren eine homoerotische Liebe hegte. Auch der Archäologe Hanold versetzt sich in seinem Traum in die Stadt Pompeji kurz vor ihrem Untergang und erblickt, wie ein Mädchen von ihm Gradiva genannt - von Asche zugeschüttet ums Leben kommt. Die junge Frau, die er jetzt in seinem Traum sieht, ist eine Gestalt von einem während seiner Forschungen entdeckten Relief, er kennt jedoch nicht die Quelle dieses Interesses. Hanolds Traum ist nach Freud sowohl eine Reminiszenz und Verzerrung des am Tage Erlebten als auch die Anfangsphase der Wiederkehr des Verdrängten. Der Archäologe interessiert sich für das Relief, weil es ihn an seine Kindheitsfreundin erinnert. Der Traum verdeutlicht ihm dies jedoch noch nicht. Hanold ist nicht imstande, das Geträumte zu deuten und sich an Zoë zu erinnern. Wie in der Nachwirkung des Traums entschließt er sich, durch einen unbewussten Trieb gesteuert, nach Italien zu reisen.

Auch Castorps Widerstand gegen das Eindringen des Unbewussten ist noch nicht vollständig gebrochen. In seinem Traum fällt der Name Hippe noch nicht, obwohl Castorp bereits weiß, ,woran und an wen sie ihn eigentlich so lebhaft erinnerte“ (MANN 1991:127). Außerdem flieht er in seinem Traum kurz nach dieser Erkenntnis vor Dr. Krokowski, der ihm nachstellt, um „Seelenzergliederung“ ${ }^{19}$ an ihm vorzunehmen. Castorps Triebhaftigkeit ist aber dermaßen stark, dass er - seinem Widerstand zum Trotz - Chauchat begehrt, wie er früher seinen Schulfreund heimlich liebte. Der Traum macht dies klar, wenn Castorp Settembrini, den Vertreter der bürgerlichen Orientierung und Störfaktor in der Liebe zu Clawdia, mit allen Kräften aus der Traumszene zu verdrängen versucht (MANN 1991:127):

19 So werden auf dem Zauberberg die von Dr. Krokowski geführten psychoanalytischen Séancen genannt. Hans Castorp findet die Idee der ,Seelenzergliederung widerlich, andererseits aber ruft sie bei ihm große Heiterkeit hervor, „, und er lachte so sehr, daß die Tränen ihm unter der Hand hervorliefen“" (MANN 1991: 18). Castorps Ausruf „Widerlich!“ (MANN 1991:17) und seine übertriebene Heiterkeit sind instinktive Abwehrreaktionen gegen die Wiederkehr des Verdrängten. 
Der Sieg der „Unform“. Das „Schnee“-Kapitel des Zauberbergs

Er bemühte sich, mit der Schulter Settembrini vom Fleck zu drängen, welcher dastand und lächelte, - fein, trocken und spöttisch, unter dem vollen schwarzen Schnurbart. [...] ,Sie stören " hörte er sich deutlich sagen. ,Fort mit Ihnen! Sie sind nur ein Drehorgelmann, und Sie stören hier!

Die Träume zeigen nun, dass Castorps Widerstand gegen Triebhaftigkeit und „asiatische“ Lässigkeit sowie sein Versuch, die bürgerliche Existenzform aufrechtzuerhalten, nichts anderes als Selbstbetrug sind. In dem Traum, den er sogar zweimal träumt, küsst Castorp von innen Madame Chauchats Schulmädchenhand. Castorp weiß noch nicht, dass diese Hand ihn in der Walpurgisnacht ins Totenreich führen wird. Er genießt beim Küssen dieser Hand die Ungezwungenheit der Unform. Chauchat gibt ihm ,,jenes Gefühl von wüster Süßigkeit“" (MANN 1991:128), das er schon vom Sitzenbleiben in der Schule kennt. ${ }^{20}$ Wie in den letzten Monaten der Schulzeit, als er schon wusste, dass er sitzenbleiben würde und sich nicht mehr, wie die anderen Schüler, anstrengen musste, genießt er in Clawdias Anwesenheit auch diese „bodenlosen Vorteile der Schande“" (MANN 1991:128).

Erst im nächsten Traum kommt das wiederkehrende Verdrängte in der Gegenwart an. Im Abschnitt „Hippe“ unternimmt Castorp alleine einen Ausflug in die Berge, wo er auf einer Bank von Pribislav Hippe träumt. Es lassen sich Parallelen zwischen diesem Traum und dem „Schnee“-Traum feststellen: In beiden Fällen begibt sich Castorp an einen abgeschiedenen Ort. In der Einsamkeit, von der Natur berauscht und durch Todesneigung verführt, hat Castorp eine Vision. Auch dieser Ausflug wird mit der Hadesfahrt verglichen. Es ist eine Fahrt in die eigene Unterwelt - in die Tiefen des Unbewussten. Der Wildbach, den Castorp überschreiten muss, steht für den Grenzfluss zum Hades, Acheron. Beim Nasenbluten fällt er ins Träumen und die Erinnerung an Pribislav Hippe wird wachgerufen. ${ }^{21}$ Das unterdrückte Gefühl

20 Der gleiche Ausdruck ist im Abschnitt „Herr Albin“ zu finden. Castorp will zur Gemeinschaft der Kranken gehören, weil er sich nach ihrer absoluten Freiheit sehnt. In seinen Gedanken versetzt er sich in Herrn Albins Zustand und stellt sich vor, „wie es sein müsse, wenn man endgültig des Druckes der Ehre ledig war und auf immer die bodenlosen Vorteile der Schande genoss." Castorp erschreckt aber dabei ,ein Gefühl von wüster Süßigkeit, das sein Herz vorübergehend zu noch hastigerem Gange erregte“ (MANN 1991:113; Hervorhebung A.J.).

21 In der Mythologie werden die Toten mit Blut aus dem Hades gelockt. Durch Blut bekommen sie Leib und Leben zurück. Bei HOMER (1970:153) begibt sich Odysseus in die Unterwelt, um den toten Propheten Teiresias nach dem Weg nach Ithaka zu fragen. Auch er wird mit Blut gerufen: „Und nachdem ich fle- 
kehrt bei Castorp wieder: „Man kann sagen, dass die Gestalt des ,Kirgisen“ unmerklich aus Nebeln in sein Leben getreten war, langsam immer mehr Deutlichkeit und Greifbarkeit gewonnen hatte, bis zu jenem Augenblick der größten Nähe und Körperlichkeit...“ (MANN 1991:168). Pribislav Hippe kehrt aus dem Unbewussten zurïck wie ein Toter, der durch Blut gestärkt, aus dem Hades zurückkommt. Wie in der Erzählung Gradiva die Reise nach Pompeji die Besessenheit der Figur betont und zum Ziel hat, das Rätsel der unterdrückten Gefühle zu lösen, indem Hanold in Gradiva Zoë erkennt, so bewirkt Castorps Ausflug ins Gebirge die Wiederkehr des Verdrängten.

Betrachtet man Träume als erfüllten Wunsch (FREUD 1973:90), kann man weitere Zusammenhänge zwischen den Träumen Castorps und der freudschen Analyse der Erzählung Gradiva feststellen. Im „Schnee“-Kapitel macht Castorp wieder einen Ausflug in die Alpen. Als er im „Schnee“-Traum den Rückweg nicht finden kann, träumt er, fast ohnmächtig, seinen Traum vom Wesen der Menschheit. Sein Traum ist erstens ein „Wiedererkennen, [...] als hätte er das blaue Sonnenglück [...] von je im Herzen getragen“ (MANN 1991:669-670). Zweitens ist er die Reminiszenz der SettembriniNaphta-Auseinandersetzung aus dem Abschnitt „Operationes spirituales“, in dem das Denken der beiden Pädagogen in Antithesen Castorp verwirrt. Auch das stimmt mit der Traumtheorie überein, denn ,ein Traum [hängt] regelmäBig mit den Tätigkeiten am Tage vor dem Traum [zusammen]" (Freud 1973:123), das heißt, „Tagesreste“ (FREUD 1973:161) liefern das Material für den Traum. Den Traum deutet Freud auch als erfüllten Wunsch des Träumenden, der sich vorwiegend dessen Seelenzustände schildert und auf die $\mathrm{Zu}$ kunft ausgerichtet ist. Auch in Castorp träumt der, Wille' einen Traum, doch dieser Traum besteht aus zwei Visionen. Neben dem Traum von der apollinischen Lichtvision erblickt Castorp die dionysische Nachwelt - das grausame Blutmahl im Tempel. Gemäß Freuds Theorie wäre auch diese grässliche Vision als verstellter Ausdruck von Castorps Wunsch zu deuten.

Schon in der ersten Nacht auf dem Zauberberg hat Castorp einen merkwürdigen Traum vom Tode. In seinen Nachtphantasien sieht er seinen Cousin Joachim Ziemßen in einer ,sonderbar verrenkte[n] Lage auf einem Bobschlitten eine schräge Bahn hinabfahren“ (MANN 1991:29). Da wegen der unbefahrbaren Wege im Winter Leichen auf Schlitten ins Tal hinuntergefahren

hend die Schar der Toten gesühnet, nahm ich die Schaf' und zerschnitt die Gurgeln über der Grube; schwarz entströmte das Blut, und aus dem Erebos kamen viele Seelen herauf der abgeschiedenen Toten.“ 
Der Sieg der „Unform“. Das „Schnee“-Kapitel des Zauberbergs

werden, kann man daraus schließen, dass Castorp von einem toten Joachim träumt. In Bezug auf Freuds Traumdeutung ist auch dieser Traum ein erfüllter Wunsch Castorps, sich dem Tode hinzugeben und selbst den Tod zu erfahren. Auch der Traum von Madame Chauchat auf dem Schulhof ist als erfüllter Wunsch zu deuten: Hier sehnt sich Castorp nach der körperlichen Verschmelzung mit Clawdia und nach der von Chauchat verkörperten Lässigkeit.

Im „Schnee“-Traum erfährt Hans Castorp die Wahrheit vom Wesen der Menschheit. Die Menschheit ist aber nicht nur das junge Sonnenglück, sondern auch das Dunkle und Grausame. In der Blutmahl-Vision erkennt Castorp seine eigene Neigung zu Tod und Unform. Einerseits ist das Blutmahl die Verkörperung des Todes und des Thanatos, andererseits die der menschlichen Triebhaftigkeit, der körperlichen Liebe und des Eros. Wie die alten Frauen im kannibalischen Ritual ein Kind zerreißen, so wird auch der Mensch von seinen unmoralischen Triebwünschen, dem ursprünglichen Prinzip und dem ,Willen` zerrissen. Die Tempel-Vision zeichnet ein düsteres Bild der Welt und des Menschen: das immer gleiche Werden und Vergehen, Fressen und Gefressenwerden, Gewalt und Verfeindung ohne Moral. Die Hexen zerreißen das Kind so, wie das stets unbefriedigt bleibende Streben, die Gier und das Verlangen das Innere des Menschen zerreißen. Glück ist hier nicht vorhanden - es ist sowohl Schopenhauers Welt des Willens als auch das dionysische Prinzip der Geburt der Tragödie.

Durch den Traum wird Castorp bewusst, dass das menschliche Leben aus diesen zwei Bereichen besteht und beide Elemente gleichzeitig vorkommen müssen. Obwohl die Gegensätze sich nicht versöhnen lassen, ist ein Gleichgewicht nötig, das als Forderung nach Lebensfreundlichkeit und Menschheitsliebe zu verstehen ist. Das, was in Castorps Traum von Bedeutung ist, ist die Erkenntnis, dass das Sonnenglück nur eine Utopie, ein Menschheitstraum und allgemeiner Wunsch ist. Dagegen gleichen die Todesneigung und das ewige Streben nach Befriedigung der Triebe dem Blutmahl im Tempel. Die Wirklichkeit besteht aus zwei Bereichen - aus der Welt des Apollinischen und des Dionysischen. Castorp entscheidet sich in seinem Traum für die Position der Mitte: die Menschlichkeit liege inmitten der Unform und der Überform, des Todes und der Vernunft, zwischen Madame Chauchat und dem Fortschrittsdenker Settembrini.

Im „bodenlosen Schweigen“ (MANN 1991:649) der Schneewelt werden Castorp das Unbewusste und das Gewünschte in Form des Traumes bewusst, 
im Gedankentraum deutet er die erfahrenen Visionen. Im Unterschied zum Archäologen Hanold, der nicht imstande war, seinen Traum zu interpretieren, übersetzt Castorp den manifesten Trauminhalt - die Sonnenleute- und Blutmahlvision - in latente Traumgedanken (FREUD 1973:134) und fasst seine Erkenntnis zusammen. Beide Träume müssen ,übersetzt“ werden, denn

[...] die Traumbilder seien die [...] psychologischen Wahnschöpfungen eines Menschen, die Kompromißergebnisse jenes Kampfes zwischen Verdrängtem und Herrschendem. [...] Die Traumbilder [hat man] als etwas Entstelltes zu betrachten, hinter dem etwas anderes, nicht Entstelltes, aber im gewissen Sinne Anstößiges zu suchen ist.

Das Charakteristische der Träume liegt in der Eliminierung der Grenzen zwischen Vergangenheit und Gegenwart. Im „Schnee“-Traum wird Castorp in die vergangene Welt zurückversetzt und erblickt nicht nur die vergangene Form des Lebens der Sonnenleute und das Grausame im Tempel. Das Vergangene erweist sich vielmehr zugleich als das Gegenwärtige und das Zukünftige. Was Castorp in seinem Traum erlebt, ist allgegenwärtig, von Raum- und Zeitrahmen unabhängig. Auch hier hat die Zeit kein absolutes Maß.

Hanolds Traum spielt sich ebenfalls nicht in der Gegenwart ab (selbst wenn er von einer Lebenden träumt), sondern im Jahr 79 n. Chr. in Pompeji beim Vesuvausbruch. In der freudschen Traumtheorie wird solch ein Verfahren die „Entstellung durch Verschiebung“ (FREUD 1973:133) genannt - nicht Gradiva wird in die Gegenwart, sondern der Träumende in die Vergangenheit versetzt. Diese Art der Entstellung wird folgendermaßen erklärt: Phantasien seien „Abkömmlinge“ verdrängter Erinnerungen, die durch die „Zensur des Widerstandes" verändert werden und entstellt ins Bewusstsein gelangen (FREUD 1973:133). Im Falle Hanolds spielt die Altertumswissenschaft die Rolle der Zensur. Die Kindheitserlebnisse mit Zoë werden verdrängt, an ihre Stelle treten „Abkömmlinge der Erinnerung“ an sie - die Phantasie über Gradiva wird zur „Wiederkehr des Verdrängten“.

Durch die „Entstellung durch Verschiebung“ wird Castorp in seinem Traum in die Welt der glücklichen Sonnenleute, in deren Nachbarschaft sich ein Blutmahl abspielt, versetzt. In der letzten Szene von Hanolds Traum verwandelt sich Gradiva in ein steinernes Relief - nach FREUD (1973:125) die poetische Darstellung seines Interesses an der Archäologie, die ihn von der Liebe zu Zoë ablenkte. Castorp erblickt dagegen in der letzten Phase seiner Vision zwei ein Kind zerreißende Weiber. Analytisch gedeutet ist das Blutmahl im 
Tempel eine poetische Darstellung der Sympathie mit dem Tod, die Castorp im Flachland immer vom Leben ablenkte.

Hanold träumt von Pompeji, denn er hat den Wunsch, Augenzeuge der Katastrophe zu sein. Hans Castorps Visionen im „Schnee“-Traum sind ebenfalls Ausdruck seiner Wünsche. Die südliche Landschaft und das Sonnenglück erscheinen als Wiedererinnerungen des seit jeher Gewussten, während die Barbarei im Tempel als die im Flachland unterdrückte Todesneigung, Triebhaftigkeit und Unform zu deuten ist. ${ }^{22}$ Erst auf dem Zauberberg, gefördert durch die leidenschaftliche Liebe zu Chauchat, die Krankheit und den Tod, kommen diese unterdrückten Sympathien zutage. Castorp ist aber beim Anblick des Blutmahls entsetzt, „es [wird] ihm so übel wie noch nie“, verzweifelt will er sich „,von der Stelle reißen“ (MANN 1991:674). Die Todessympathie, die er so idealisiert und romantisiert hat, erschreckt ihn, als die alten Frauen „die blutigen Fäuste nach ihm“ reckten (MANN 1991:674). Er erkennt die wahre Gefahr seiner eigenen Neigungen, was ihn wiederum zur Antwort auf die Frage nach dem Leben bringt - die Antwort, die er in seinen Studien so lange vergeblich suchte, und die sich zuletzt auf die einfache Frage, was der Mensch sei, zurückführen lässt. Deswegen nimmt er im Gedankentraum Abschied von seinen früheren Überzeugungen, d.h. von der Meinung, die er noch vor den Erlebnissen der Walpurgisnacht vertreten hatte, dass „,man sich namentlich für den Tod“ interessiere, ,,wenn man sich für das

22 Dass Castorp seine Neigungen im bürgerlichen Leben des Flachlandes verdrängt, zeigt auch Kristiansen in seinen Studien zu den Leitmotiven im Zauberberg. Die verdrängten, unbewussten Neigungen sind in zahlreichen Motiven zu finden: in Castorps Vorliebe für Musik, Freizeit und Freiheit sowie in seinen bereits erwähnten ,nicht sonderlich aristokratische[n] Händen“ (MANN 1991:46), deren Mängel Hans durch Pflege zu verhüllen versucht. Das ist sein Versuch, die Neigung zu Lässigkeit und Unform in der bürgerlichen Welt zu verdrängen. Die Leitmotivstruktur zeigt, dass das Sexuelle eine thematische Einheit mit Tod und Krankheit bildet, so dass Castorp ein problematisches Verhältnis zum Sexuellen hat, wenn sein Verhältnis zum Tod problematisch ist. Er unterdrückt also im Flachland sowohl das Triebhafte als auch die Todesneigung. Erst der Aufenthalt auf dem Zauberberg fördert die Wiederkehr des Verdrängten, die sich durch seine Febrilität, seine Liebe zu Chauchat und letztlich durch den Sieg der Unform über die bürgerlichen Normen ausdrückt. Die Blutmahl-Vision ist also nicht nur die Veranschaulichung des Todes, sondern auch die Darstellung der einen Menschen zerreißenden Triebe und dunklen Mächte, die Castorp zusammen mit der Erinnerung an Pribislav Hippe zu verdrängen suchte. 
Leben interessiert“ (MANN 1991:366). Die Schlüsselerkenntnis dieses Traumes lautet nun (MANN 1991:677):

Ich will dem Tode Treue halten in meinem Herzen, doch mich hell erinnern, daß Treue zum Tode und Gewesenen nur Bosheit und finstere Wollust und Menschenfeindschaft ist, bestimmt sie unser Denken und Regieren. Der Mensch soll um der Güte und Liebe willen dem Tode keine Herrschaft einräumen über seine Gedanken.

Für Castorp bedeutet also der Gedankentraum einerseits die Überwindung seiner im Flachland unterdrückten und auf dem Zauberberg wiedergekehrten Neigungen im Traum. Andererseits liefert der Traum Erlebnisse, die Castorp an das Humanitätsproblem denken lassen. Die Schwäche dieser Liebe besteht darin, dass sie lediglich ein Traumprodukt ist. Castorp versucht zwar im weiteren Teil des Romans, die humane Idee der Unform seinen eigenen Neigungen gegenüberzustellen, vermag aber die neue Einsicht nicht zu realisieren. Schließlich ergibt er sich dem Stumpfsinn und verfällt endgültig der Unform.

\section{„Schnee“-Reminiszenzen}

Das „Schnee“-Kapitel endet mit einem Satz, der in der Forschung oft als das definitive Auslöschen der Erinnerung gesehen wird: „Was er gedacht, verstand er schon diesen Abend nicht mehr so recht“ (MANN 1991:679). Nach dem Erwachen tritt Castorp den Rückweg an, und noch bevor er im Sanatorium ankommt, besucht er Settembrini. In der letzten Szene des Abschnittes macht der Erzähler einen weiteren Kommentar: „Die hochzivilisierte Atmosphäre des ,Berghofs“ umschmeichelte ihn eine Stunde später. Beim Diner griff er gewaltig zu. Was er geträumt, war im Verbleichen begriffen." (MANN 1991:679) Diese doppelte Bemerkung des Erzählers sollte aber nicht als absolutes Vergessen gedeutet werden, denn im weiteren Teil des Romans gibt es mehrfach Stellen, die für das Nicht-Vergessen des Traums sprechen. Castorp erinnert sich an das „Schnee“-Erlebnis und befindet sich im Konflikt zwischen Todesneigung und humaner Idee, die er aus seinem Gedankentraum kennt. Dies soll nun im Einzelnen gezeigt werden.

Nicht nur an dieser Stelle im Roman ,vergisst ' Castorp ausschlaggebende Momente in seinem Leben. In der Episode mit Pribislav Hippe kommt das ,Vergessen“ zum ersten Mal vor. Wegen der Versetzung seines Vaters verlässt Hippe Schule und Stadt, ,,aber das beachtete Hans Castorp kaum noch, er hatte ihn schon vorher vergessen“ (MANN 1991:168) - fügt der Erzähler 
auktorial hinzu. Auch dieses Vergessen ist, wie die Analyse von Castorps Träumen zeigte, nicht im wörtlichen Sinne zu verstehen. In der Tat verdrängt Castorp sein homophiles Gefühl, das als moralwidriger Triebwunsch dargestellt wird. Als Symbol der Verdrängung gelten die Schnitzel aus Pribislavs Bleistift, die Castorp jahrelang in seiner Schublade aufbewahrt und an die er sich in dem Moment erinnert, als er den Zusammenhang zwischen Chauchat und Hippe feststellt (MANN 1991:170-171):

Es war ganz Pribislav, wie er leibte und lebte. Ich hätte nicht gedacht, daß ich ihn je so deutlich wiedersehen würde. Wie merkwürdig ähnlich er ihr sah, - dieser hier oben! Darum also interessierte ich mich so für sie? Oder vielleicht auch: habe ich mich darum so für ihn interessiert?

In diesem bewusstseinstranszendenten Zustand werden die Zeitgrenzen aufgehoben - die Gegenwart wird Vergangenheit und Vergangenheit Zukunft. Die Schublade, in der Castorp die Bleistiftschnitzel bewahrt, versinnbildlicht das Unbewusste, in dem die verdrängten Erinnerungen, die immer ,herausgeholt` werden können, versteckt bleiben.

Auch den „Schnee“-Traum vergisst Castorp nicht ganz. Dass er sich auf dem Rückweg noch mit Settembrini trifft und ihm nicht von seinem Erlebnis erzählt, bleibt für die Deutung des Vergessens nicht ohne Bedeutung. Castorps Erkenntnis liegt nicht im Bereich des Fortschrittsdenkens Settembrinis, sie stimmt mit dessen Auffassung des Humanitätsgedankens nicht überein. Wenn Castorp den Traum verheimlicht, so ist das als Ausdruck seiner Autonomie und Selbständigkeit zu deuten. Hans Castorp wird die Erkenntnis aus dem Traum, trotz der Bemerkung des Erzählers am Kapitelende, nicht vergessen, denn der Traum vergegenwärtigt ihm nur, was er „für immer weiß“ (MANN 1991:677). Dass sich Castorp an die humanistische „Mitte“ aus dem Gedankentraum erinnert, davon zeugen seine Überlegungen aus dem Abschnitt „Als Soldat und brav“ bezüglich Chauchats Reise nach Spanien, wo es zur Konfrontation der Gegensätze des „Spanischen“ und des „Asiatischen“ (KRISTIANSEN 1986:33) kommt (MANN 1991:688):

Spanien, das liege andererseits ebenso weit von der humanistischen Mitte ab, nicht nach der weichen, sondern nach der harten Seite; es sei nicht Formlosigkeit, sondern Überform, der Tod als Form, sozusagen, nicht Todesauflösung, sondern Todesstrenge, schwarz, vornehm und blutig, Inquisition, gestärkte Halskrause, Loyola, Eskorial... Interessant, wie es Frau Chauchat in Spanien gefallen werde. Das Türenwerfen werde ihr dort wohl vergehen, und vielleicht könne eine gewisse Kompensation der beiden außerhumanistischen Lager zum Menschlichen sich vollziehen. 
Die Gefährdung der humanen „Mitte“ ist nach Castorp sowohl die spanische Strenge als auch die asiatische Formlosigkeit der Madame Chauchat. Aus der Konfrontation der beiden Lager kann aber auch „etwas recht boshaft Terroristisches zustande kommen, wenn der Osten nach Spanien gehe“ (MANN 1991:688). In der „Mitte“ zwischen den beiden Todesauffassungen - der Todesstrenge und der Todessauflösung - liegt der Humanismus, die Menschheits- und Lebensliebe, die um das wahre Wesen des Todes weiß. Die Erinnerungen aus dem „Schnee“-Kapitel werden auch während der nächsten Auseinandersetzung zwischen Settembrini und Naphta zutage gefördert (MANN 1991:713):

Alles stellten sie auf die Spitze, diese zwei, wie es wohl nötig war, wenn man streiten wolle, und haderten erbittert um äußerste Wahnfälle, während ihm doch schien, als ob irgendwo inmitten zwischen den strittigen Unleidlichkeiten, zwischen rednerischem Humanismus und analphabetischer Barbarei das gelesen sein müsse, was man als das Menschliche und Humane versöhnlich ansprechen durfte.

Castorps Einstellung zu seinen Lehrmeistern ändert sich nach dem „Schnee“Traum wesentlich: Er distanziert sich von ihnen und nennt sie die „Schwätzer" (MANN 1991:676). Auch wenn er ihnen intellektuell nicht gewachsen ist, bleibt er ihnen gegenüber, wie im „Schnee“-Traum beschlossen, zurückhaltend und skeptisch. Die Negation der nicht miteinander zu vereinbarenden Lebenshaltungen der Pädagogen ist ein Beleg dafür, dass Castorp seinen Traum nicht vergisst. Er ist zwar nicht imstande, seine humane Idee dem Radikalismus der Mentoren gegenüberzustellen, hegt in sich aber zumindest eine gewisse Abneigung ihnen gegenüber, wie es ihn der „Schnee“-Traum lehrte.

Der Gedankentraum mit seinem Entwurf einer dem Tod entgegensetzten Liebe trägt auch im Verhältnis Castorps zu Chauchat und Peeperkorn Früchte. So schließt er einerseits mit der Russin ein Bündnis für den das Leben verkörpernden Peeperkorn (MANN 1991:819) und andererseits mit Peeperkorn einen mentalen Bund zugunsten der für Krankheit und Tod stehenden Clawdia (MANN 1991:838). Ihre Beziehung ist die Zusammenstellung von Gegensätzen. Castorps Ausdruck der Versöhnung dieser Polarität ist auch hier die Position der „Mitte“, die er zwischen beiden einnimmt. Er drückt damit noch einmal die Erkenntnis aus dem „Schnee“-Traum aus - Lebensbejahung sei ohne die Anerkennung des Wesens des Todes unzulänglich. Nur die Lebensfreundlichkeit, die vom Tode weiß, ist genial. Dieses Bekenntnis wiederholt Castorp im Gespräch mit Clawdia (MANN 1991:816): „die Liebe [zum Tod] führt zur Liebe des Lebens und des Menschen. [...] Zum Leben 
Der Sieg der „Unform“. Das „Schnee“-Kapitel des Zauberbergs

gibt es zwei Wege: Der eine ist der gewöhnliche, direkte und brave. Der andere ist schlimm, er führt über den Tod, und das ist der geniale Weg!“ Dieser aus dem „Schnee“-Erlebnis übernommene Gedanke ist die Korrektur seiner früheren Ansichten. Man interessiere sich für das Leben, wenn man sich für den Tod interessiere - dieses Bekenntnis ist der Ausdruck von Castorps Sympathie für das Leben. Die Bedeutung dieser Aussage wird dadurch gesteigert, dass Castorp seine Erkenntnis in Clawdias Anwesenheit wiederholt, die sie für „mähnschlich“ (MANN 1991:816) hält. „Die Menschlichkeit fängt an, wo ungeniale Leute glauben, daß sie aufhört.“ (MANN 1991:817) - konstatiert Castorp wenig später.

Diese Lebensfreundlichkeit, die vom Tode weiß, ist Castorps Ausdruck der humanen Idee. Sie ist allerdings seine private Angelegenheit, die für ihn und die Gesellschaft ohne Folgen bleibt. Die „Abkömmlinge“ des Traumes reduzieren die Neigung Castorps zur Unform und seine Sympathie für den Tod zum schlechten Gewissen, veranlassen ihn aber nicht zu entscheidenden Taten, bis sie immer schwächer werden und Castorp dem ,großen Stumpfsinn“ (MANN 1991:856) verfällt. Den Entwicklungsweg des Protagonisten brachte Neumann (2001:78) wie folgt auf den Punkt: Der Bildungsroman gehe am Ende in den Zeitroman über, so dass die Hauptfigur ihre Einsichten nicht verwirklichen kann. In ihrem Bildungsweg sei ,allenfalls ein Erbe formuliert, über dessen Annahme eine Epoche zu entscheiden hat, deren Konturen sich im Zauberberg noch nicht abzeichnen.

\section{Resümee}

Wie dieser Analyse zu entnehmen ist, lässt sich der Kern des Zauberbergs psychoanalytisch betrachten. In den Träumen Castorps sind die Grundkonzepte der Psychoanalyse zu finden - Castorp träumt mit allen typischen Erscheinungen: Tagesrest, Verdichtung und Verschiebung. Auch schildern diese Träume die Seelenzustände des Helden und fördern die Wiederkehr des Verdrängten. Die Erinnerung an Hippe ist gleichzeitig die Erinnerung an die unterdrückten Gefühle, die in Castorps Unterbewusstsein vorhanden waren und die sein Verhältnis zum bürgerlichen Leben immer bestimmten. Aber Castorp hat viel mehr zu verhüllen als nur die Kindheitsliebe. In ihm kehrt der gesamte Komplex der Neigungen und Wünsche wieder, denen er die humane Idee aus dem „Schnee“-Traum vergeblich gegenüberzustellen versucht. Die Analyse der Träume unter psychoanalytischem Gesichtspunkt erklärt die Ambivalenz der Gefühle des Protagonisten. Seine Träume zeigen, dass sein 
Widerstand gegen die Unform nichts anderes als Selbstbetrug ist. Von diesem Traum-Komplex unterscheidet sich der Schnee-Traum. Zwar gelangt Castorp durch ihn zu einer Erkenntnis, die ihn vom Bereich der Unform loszulösen scheint, doch letzten Endes erweist sie sich als abstrakte und folgenlose Meinung. Dass Castorp seinen Traum nicht vergisst, zeigen die Reminiszenzen des Traumes, auch zum Teil fast wörtliche Wiederaufnahmen der Einsichten des „Schnee“-Kapitels. Sie werden im weiteren Teil des Romans mehrmals aufgegriffen. Castorp erinnert sich an das „Schnee“-Erlebnis und befindet sich im Konflikt zwischen Todesneigung und humaner Idee, die er aus seinem Gedankentraum kennt. Die Handlung des Zauberbergs ist nach dem „Schnee“-Traum der ständige Kampf der Neigungen des Protagonisten gegen die geträumte humane Idee. Castorp besiegt seinen Drang zur Unform nicht. Die Abwehrreaktionen vor den eigenen Neigungen werden immer schwächer, bis Hans Castorp endgültig der Unform verfällt.

\section{Literatur}

DiERKS, MANFRED (1991): Traumzeit und Verdichtung. Der Einfluß der Psychoanalyse auf Thomas Manns Erzählweise. In: HeFtrich, ECKHARD / KOOPMANN, HeLmut (eds.): Thomas Mann und seine Quellen. Festschrift für Hans Wysling. Frankfurt (M.), 111-137.

DIERSEN, Inge (1965): Untersuchungen zu Thomas Mann. Berlin.

ERKME, JoSEPH (1998): Nietzsche im „Zauberberg “. Frankfurt (M.) (=Thomas-MannStudien 14).

Freud, Sigmund (1973): Der Wahn und die Träume in W. Jensens „Gradiva“ mit dem Text der Erzählung von Wilhelm Jensen. Hrsg. von Bernhard Urban und Johannes Cremerius. Frankfurt (M.), 87-161.

- (1992): Das Unbewußte. In: Das Ich und das Es - Metapsychologische Schriften. Frankfurt (M.), 117-153.

Hick, Christian (2003): Vom Schwindel ewiger Gegenwart. Zur Pathologie der Zeit in Thomas Manns „Zauberberg“. In: ENGELHARDT, DIETRICH VON / WISSKIRCHEN, HANS (eds.): „Der Zauberberg“ - die Welt der Wissenschaften in Thomas Manns Roman. Stuttgart/New York, 71-106.

HiLSCHER, EBERHARD (1968): Thomas Mann. Sein Leben und Werk. Berlin.

HOMER (1970): Odyssee. Stuttgart.

JendREIECK, Helmut (1977): Thomas Mann. Der demokratische Roman. Düsseldorf.

Koopmann, Helmut (1988): Philosophischer Roman oder romanhafte Philosophie. $\mathrm{Zu}$ Thomas Manns lebensphilosophischer Orientierung in den zwanziger Jahren. In: WolfF, Rudolf (ed.): Thomas Mann. Aufsätze zum „Zauberberg“. Bonn, 61-88. 
Der Sieg der „Unform“. Das „Schnee“-Kapitel des Zauberbergs

- (ed.) (1995): Thomas-Mann-Handbuch. Stuttgart.

KRISTIANSEN, BøRGE (1985): „Der Zauberberg “: Schopenhauer-Kritik oder Schopenhauer-Affirmation? In: KURZKE, HERMANN (ed.): Stationen der Thomas-Mann-Forschung. Aufsätze seit 1970. Würzburg, 135-144.

- (1986): Thomas Manns „Zauberberg“ und Schopenhauers Metaphysik. Bonn.

KuRZKe, Hermann (1985): Thomas Mann. Epoche - Werk - Wirkung. München.

- (2001): Thomas Mann. Das Leben als Kunstwerk. Eine Biographie. München.

LANGer, Daniela (2007): „was ich Humanität nenne“: Zur Kontinuität des Bildungsbegriffs bei Thomas Mann vom Ersten Weltkrieg bis zur jungen Weimarer Republik. In: WitT, MONIKa / KUnicKI, WOJCIECH (eds.): Bildung. Facetten der wissenschaftichen Kommunikation. Nysa/Wrocław, 387-398.

- (2009): Erläuterungen und Dokumente zu: Thomas Mann: „Der Zauberberg“. Stuttgart.

LEHNERT, HERBERT (1960): Hans Castorps Vision. Eine Studie zum Aufbau von Thomas Manns Roman „Zauberberg“. The Rice Institute Pamphlet 47:1-37.

MAAR, MichAEL (1995): Geister und Kunst. Neuigkeiten aus dem Zauberberg. München.

Mádl, Antal (1980): Thomas Manns Humanismus. Wende und Wandel einer Weltund Menschenauffassung. Berlin.

ManN, Thomas (1960): Von deutscher Republik. In: Gesammelte Werke in zwölf Bänden. Bd. 11. Frankfurt (M.), 809-852.

- (1962): Briefe 1889-1936. Bd.1. Hrsg. von Erika Mann. Frankfurt (M.).

- (1974): Einführung in den „Zauberberg “. Für Studenten der Universität Princeton. In: Gesammelte Werke in dreizehn Bänden. Bd. 11. Frankfurt (M.), 602-617.

- (1991): Der Zauberberg. Frankfurt (M.).

Mertens, VolKer (2006): Groß ist das Geheimnis. Thomas Mann und die Musik. Leipzig.

Neumann, Michael (2001): Die Irritationen des Janus oder „Der Zauberberg“ im Feld der klassischen Moderne. In: Thomas-Mann-Jahrbuch 14:69-85.

- (2002): Kommentar zu: Thomas Mann: „Der Zauberberg. Roman“. Frankfurt (M).

NiETZSCHe, FriedRICH (1980): Zur Genealogie der Moral. In: Sämtliche Werke. Kritische Studienausgabe in 15 Bänden. Hrsg. von Giorgio Colli und Mazzino Montinari. Bd. 5. München.

- (1999): Die Geburt der Tragödie aus dem Geiste der Musik. München.

Pütz, Peter (1978): Thomas Mann und Nietzsche. In: Hillebrand, Bruno (ed.): Nietzsche und die deutsche Literatur. Tübingen, 121-154.

- (1995): Krankheit als Stimulans des Lebens. Nietzsche auf dem Zauberberg. In: SPRECHER, 249-264.

- (1999): Nachwort. In: NIETZSCHE, 160-188. 
Aneta Jachimowicz

RIECKMANN, JENS (1979): „Der Zauberberg“: Eine geistige Autobiographie Thomas Manns. Stuttgart.

SCHARFSCHWERDT, JÜRGEN (1967): Thomas Mann und der deutsche Bildungsroman. Stuttgart.

SCHOPENHAUER, ARTHUR (1961): Versuch über das Geistersehen und was damit zusammenhängt. In: Parapsychologische Schriften. Hrsg. von Hans Bender. Basel, 95-206.

SPRECHER, ThOMAS (ed.): Das Zauberberg-Symposium 1994 in Davos. Frankfurt (M.) (=Thomas-Mann-Studien 11), 249-264.

Urban, Bernhard / Cremerius, Johannes (1973): Einleitung. In: Freud, 7-20.

Wysling, Hans (1988): Probleme der „Zauberberg“-Interpretation. In: ThomasMann-Jahrbuch 1:12-26.

- (1995): „Der Zauberberg“. In: Koopmann, Helmut (ed.): Thomas-Mann-Handbuch. Stuttgart, 397-422.

- (1995a): „Der Zauberberg “ - als Zauberberg. In: SPRECHER, 43-57.

Wysling, Hans / Fischer, MaRIANNE (1975): Dichter über ihre Dichtungen. Bd. 1. Zürich/München/Frankfurt (M.). 